\title{
GEOMETRY OF THE SHILOV BOUNDARY OF A BOUNDED SYMMETRIC DOMAIN
}

\author{
JEAN-LOUIS CLERC
}

Communicated by Ivaïlo M. Mladenov

\begin{abstract}
In the first part, the theory of bounded symmetric domains is presented along two main approaches: as special cases of Riemannian symmetric spaces of the noncompact type on one hand, as unit balls in positive Hermitian Jordan triple systems on the other hand. In the second part, an invariant for triples in the Shilov boundary of such a domain is constructed. It generalizes an invariant constructed by E. Cartan for the unit sphere in $\mathbb{C}^{2}$ and also the triple Maslov index on the Lagrangian manifold.
\end{abstract}

\section{Contents}

1. Introduction 2

2. Hermitian Symmetric Spaces $\quad \mathbf{2 8}$

2.1. Riemannian Symmetric Spaces . . . . . . . . . . . . . . . . . 28

2.2. Hermitian Symmetric Spaces . . . . . . . . . . . . . . . . . . 31

2.3. The Harish Chandra Embedding . . . . . . . . . . . . . . . . . . . . . 32

2.4. Jordan Triple System . . . . . . . . . . . . . . . . . . . . . . . . . . . 33

2.5. An Example: the Hermitian Symmetric Space of Type $\boldsymbol{I}_{\boldsymbol{p}, \boldsymbol{q}} \ldots \ldots \ldots$

3. Bounded Symmetric Domains 38

3.1. Bergman Metric . . . . . . . . . . . . . . . . . . . . 38

3.2. Bounded Symmetric Domains . . . . . . . . . . . . . . . . . . . 39

3.3. The Spectral Norm on $\mathbb{E} \ldots \ldots$. . . . . . . . . . . . . . . . . . 41

3.4. An Example (Continued from Section 2.5) . . . . . . . . . . . . . . . . 42

4. The Shilov Boundary of a Bounded Symmetric Domain 45

4.1. The Shilov Boundary of a Bounded Domain . . . . . . . . . . . . . 45

4.2. More on Tripotents . . . . . . . . . . . . . . . . . . . . . 46

4.3. Geometry of the Convex Set $\overline{\mathcal{D}} \ldots \ldots \ldots$. . . . . . . . . . . 48

4.4. The Shilov Boundary of $\mathcal{D} \ldots \ldots$. . . . . . . . . . . . . . . . 49

4.5. The Arithmetic Distance and the $G$-orbits in $S \times S \ldots \ldots$

4.6. Example (Continued from Sections 2.5 and 3.4) . . . . . . . . . . . 51

5. Euclidean Jordan Algebras and Tube-type Domains 53

5.1. Euclidean Jordan Algebra . . . . . . . . . . . . . . . . . . 53

5.2. Hermitification of a Euclidean Jordan Algebra . . . . . . . . . . . . . 55 
5.3. Euclidean Jordan Algebra Associated to a Tripotent _ . . . . . . . . . 56

5.4. Euclidean Jordan Algebras vs PHJTS . . . . . . . . . . . . . . . . . 57

5.5. The Cayley Transform . . . . . . . . . . . . . . . . . . 57

6. The Triple Invariant $\quad 59$

6.1. The Symplectic Area of a Geodesic Triangle . . . . . . . . . . . . . . . . 59

6.2. The Limit Process . . . . . . . . . . . . . . . . . . . . . . . 61

6.3. Example: Elie Cartan's Invariant . . . . . . . . . . . . . . . . . 63

6.4. Example: the Triple Maslov Index . . . . . . . . . . . . . . . . . . 64

7. G-orbits in $S \times S \times S$ (Tube-type Case) 66

8. The Maslov Index for Paths 68

9. Appendix: List of Bounded Symmetric Domains and their Shilov Boundaries 71

10. References

\section{Introduction}

The present paper is an outgrowth of the cycle of conferences delivred by the author at the Tenth International Conference on Geometry, Integrability and Quantization, held in Varna in June 2008. The first part (sections 2-5) is a survey of the theory of bounded symmetric domains. Since their introduction by E. Cartan, bounded symmetric domains have been intensively studied. There are two main trends to present them. The usual approach first studies Hermitian symmetric spaces as special cases of Riemannian symmetric spaces, namely those which admit a compatible complex structure. For the noncompact type, the theory culminates with the Harish Chandra embedding theorem, which realizes the space as a bounded symmetric domain in $\mathfrak{P}^{+}$, where $\mathfrak{P}^{+}$is the holomorphic tangent space at some (any) point of the space. The bounded domain thus obtained in $\mathfrak{P}^{+}$ is circled and can be characterized by a norm condition (see Theorem 11) .

The second approach starts with a bounded circled symmetric domain $\mathcal{D}$ in some complex vector space $\mathbb{V}$ and shows that the Lie algebra of vector fields generated by the group of holomorphic diffeomorphisms of $\mathcal{D}$ has a very specific realization, which induces on $\mathbb{V}$ a rich algebraic structure (positive Hermitian Jordan triple system, PHJTS for short). Conversely, for each positive Hermitian Jordan triple system $\mathbb{V}$, it is possible to develop a spectral theory for the elements of $\mathbb{V}$ and in particular to define a certain complex Banach norm on $\mathbb{V}$ called the spectral norm. The unit ball for the spectral norm can be shown to be a bounded symmetric domain. The Bergman metric (which exists for any bounded domain) realizes the bounded symmetric domain as a Hermitian symmetric space of the noncompact type. This approach shows a one to one correspondance between PHJTS and bounded symmetric domains, which in a sense, shows that the concept of PHJTS 
is exactly fitted for the study of bounded symmetric domains.

The characterization of the Shilov boundary of a bounded symmetric domain is specially nice in the approach through PHJTS, as the elements of the Shilov boundary can be characterized by an algebraic property (they are the maximal tripotents).

There is an important difference inside bounded symmetric domains: some of them are said to be of tube-type, the others being not of tube-type. The Cayley transform (a generalization of the classical Cayley transform mapping the open unit disc in $\mathbb{C}$ into the upper half-plane $\operatorname{Im} z>0$ ) transforms a domain of tube type in a Siegel domains of type I, which is a generalized half-space over a convex cone, whereas a non tube type domain is transformed into a Siegel domain of type II. Tube-type domains correspond to a special class of PHJTS, namely those which are obtained from a Euclidean Jordan algebra by the process of Hermitification (a variant of the complexification). The interplay between the two notions (tube type vs non-tube type, PHJTS vs Euclidean Jordan algebra) is an important tool in studying fine properties of bounded symmetric domains.

The second part (sections 6-8) is a presentation of some of the results obtained during the last years by the author, partially in collaboration with B. Ørsted, K-H. Neeb and K. Koufany (see 4-11). The main theme is to diffeomorphisms group $G$ of a bounded symmetric domain $\mathcal{D}$ on triplets in the Shilov boundary $S$ of $\mathcal{D}$. An invariant is constructed for this action. This invariant coincides with known invariants in specific cases. The classical triple Maslov index, a $\mathbb{Z}$-valued invariant (for the symplectic group) on triples of Lagrangians is the most famous. For tube type domains, this triple invariant is the main ingredient in the classification of orbits of $G$ into $S \times S \times S$.

To help the reader, an example (the unit ball in the space of complex rectangular matrices) is followed through the paper (see Sections 2.5, 3.4 and 4.6), and most of the concepts that are introduced in a general setting are explicitely determined for this example. Somme other examples appear occasionally.

The paper contains no proof (except for a few proofs that are sketched) but I tried to give appropriate references. In preparing these notes, I used three main sources: the classical treatise by S. Helgason [17] and specially chapter VIII, the book by Satake [30] which combines the classical approach and the use of Jordan triple system, and the notes by O. Loos [25], where Jordan triple systems are the main tool for studying bounded symmetric domains. The books [17] and [30] contain many references to the literature on the subject. 


\section{Hermitian Symmetric Spaces}

\subsection{Riemannian Symmetric Spaces}

The basic reference for this section is Helgason's book [17]. For a different point of view, see [24].

Definition 1. A connected Riemannian manifold $(M, g)$ is a Riemannian symmetric space if, for each point $m \in M$, there exists an involutive isometry $s_{m}$ of $(M, g)$ such that $m$ is an isolated fixed point of $s_{m}$.

The differential $D s_{m}(m)$ of $s_{m}$ at $m$ is an involution of the tangent space $T_{m} M$, and, because $m$ is an isolated fixed point, 1 can not be an eigenvalue of $D s_{m}(m)$. Hence $D s_{m}(m)=-\mathrm{id}_{T_{m} M}$, so that $s_{m}$ has to coincide with the geodesic symmetry around $m$ (a priori only locally defined, and not necessarily locally isometric). If there is an isometry $s_{m}$ satisfying the requirements of the definition, then it is unique and called the geodesic symmetry centered at $m$.

For a general Riemannian manifold $M$, the group $\operatorname{Is}(M)$ of isometries of $(M, g)$ with the compact-open topology has a unique compatible structure of Lie group (Myers-Steenrod theorem, see [28]). When $M$ is a Riemannian symmetric space, composition of symmetries centered at various points of $M$ produces enough isometries of $M$ to prove that the group $\operatorname{Is}(M)$ is transitive on $M$. A refinement says that the same statement is true for the neutral component of $\operatorname{Is}(M)$. We will denote by $G$ the neutral component of $\operatorname{Is}(M)$.

Fix an origin $o$ in $M$. Let $K$ be the isotropy subgroup of $o$ in $G$. Then $K$ is a closed compact subgroup of $G$, and $M$ is isomorphic to the quotient space $G / K$. Let $\mathfrak{g}=$ Lie $(G)$ be the Lie algebra of $G$, and $\mathfrak{k}$ the Lie algebra of $K$ viewed as a Lie subalgebra of $\mathfrak{g}$. The tangent space $T_{o} M$ of $M$ at $o$ can be identified with $\mathfrak{g} / \mathfrak{k}$. The map

$$
\theta: G \longrightarrow G, \quad g \longmapsto s_{o} \circ g \circ s_{o}
$$

is an involutive automorphism of $G$. Let $G^{\theta}=\{g \in G ; \theta(g)=g\}$ be the fixed points set of $\theta$. Then $G^{\theta}$ is a compact subgroup of $G$, and

$$
\left(G^{\theta}\right)_{0} \subset K \subset G^{\theta} .
$$

The differential of $\theta$ at the identity is an involutive automorphism of the Lie algebra $\mathfrak{g}$, still denoted by $\theta$. There is a corresponding decomposition

$$
\mathfrak{g}=\mathfrak{k} \oplus \mathfrak{p}
$$


where

$$
\mathfrak{k}=\{X \in \mathfrak{g} ; \theta X=X\}, \quad \mathfrak{p}=\{X \in \mathfrak{g} ; \theta X=-X\} .
$$

Moreover,

$$
[\mathfrak{k}, \mathfrak{k}] \subset \mathfrak{k}, \quad[\mathfrak{k}, \mathfrak{p}] \subset \mathfrak{p}, \quad[\mathfrak{p}, \mathfrak{p}] \subset \mathfrak{k} .
$$

The projection from $\mathfrak{g}$ to $\mathfrak{p}$ along $\mathfrak{k}$ yields an isomorphism of $\mathfrak{g} / \mathfrak{k}$ with $\mathfrak{p}$, and hence a natural identification $T_{o} M \simeq \mathfrak{p}$. Moreover, the map

$$
K \longrightarrow \operatorname{Hom}\left(T_{o} M\right), \quad k \longmapsto D k(o)
$$

defines an action of $K$ on $T_{o} M$, whereas $K$ acts on $\mathfrak{p}$ by the adjoint action. The isomorphism $T_{o} M \simeq \mathfrak{p}$ is equivariant with respect to these actions of $K$.

The vector space $\mathfrak{p}$ is naturally equipped with a Lie triple product (LTS) defined by

$$
[X, Y, Z]=[[X, Y], Z]
$$

Proposition 2. The Lie triple product on $\mathfrak{p}$ satisfies the following identities

$$
\begin{gathered}
{[X, Y, Z]=-[Y, X, Z]} \\
{[X, Y, Z]+[Y, Z, X]+[Z, X, Y]=0} \\
{[U, V[X, Y, Z]]=[[U, V, X], Y, Z]+[X,[U, V, Y], Z]+[X, Y,[U, V, Z]]}
\end{gathered}
$$

for all $X, Y, Z, U, V$ in $\mathfrak{p}$.

This Lie triple product has a nice geometric interpretation, namely

$$
R_{o}(X, Y) Z=-[[X, Y], Z]=-[X, Y, Z]
$$

where $R_{o}$ is the curvature tensor of $M$ at $o$ (see [17] p. 215).

The Ricci curvature (also called the Ricciform) is the symmetric bilinear form on $T_{o} M$ given by

$$
r_{o}(X, Y)=-\operatorname{Tr}\left(Z \longmapsto R_{o}(X, Z) Y\right)
$$

Proposition 3. The Ricci curvature at o is invariant under the action of $K$ on $T_{o} M$ and satisfies, for all $X, Y$ in $\mathfrak{p}$

$$
r_{o}(X, Y)=-\frac{1}{2} B(X, Y)
$$

where $B(X, Y)=\operatorname{Tr}_{\mathfrak{g}}(\operatorname{ad} X \operatorname{ad} Y)$ is the Killing form of the Lie algebra $\mathfrak{g}$. 
See [30], p. 75 for a proof.

A Riemannian symmetric space $M \simeq G / K$ is said to be of Euclidean type if $[\mathfrak{p}, \mathfrak{p}]=0$. The space $M$ is said to be irreducible if it is not Euclidean and the representation of $K$ on the tangent space $T_{o} M \simeq \mathfrak{p}$ is irreducible (admits no $K$-invariant subspace except $\{0\}$ and $\mathfrak{p})$.

If $M$ is irreducible, then there exists a unique (up to a positive real number) $K$ invariant inner product on $\mathfrak{p}$, and hence the Ricci form $r_{o}$ has to be proportional to it.

Definition 4. An irreducible Riemannian symmetric space is said to be

- of the compact type - if $r_{o}$ is positive definite

- of the noncompact type - if $r_{o}$ is negative definite.

The definition does not depend of the choice of the origin, as the group $G$ is transitive on $M$.

Any simply connected Riemannian symmetric space $M$ is a product of a Euclidean space and of irreducible symmetric spaces (see [17], Chapter V, Proposition 4.2 and Chapter VIII, Proposition 5.5). If all factors are of the compact (respectively noncompact) type, then $M$ is said to be of the compact (respectively noncompact) type.

If $M$ is of compact type, then $G$ is a compact semisimple Lie group. If $M$ is of the noncompact type, then $G$ is a semisimple Lie group (with no compact factors) and $\theta$ is a Cartan involution of $G$ (see [17], Chapter V).

For a Riemannian symmetric space of the noncompact type, the infinitesimal data characterize the space. More precisely, given a semisimple Lie algebra $\mathfrak{g}$ with no compact factors, let $G$ be any connected Lie group with Lie algebra Lie $(G)=\mathfrak{g}$ and assume that $G$ has a finite center (there always exists such a group). Let $\theta$ be a Cartan involution of $\mathfrak{g}$ (notice that two Cartan involutions of $\mathfrak{g}$ are conjugate under the adjoint action of $G$ ). Let $\mathfrak{g}=\mathfrak{k} \oplus \mathfrak{p}$ be the corresponding Cartan decomposition of $\mathfrak{g}$. The Killing form $B$ of $\mathfrak{g}$ is negative definite on $\mathfrak{k}$ and positive definite on $\mathfrak{p}$. The involution $\theta$ can be lifted to an involutive automorphism of $G$, still denoted by $\theta$. Then $K=G^{\theta}$ is a connected compact Lie subgroup of $G$. Let $M=$ $G / K$, and set $o=e K$. The tangent space at $o$ is naturally isomorphic to $\mathfrak{p}$ and $B_{\mid \mathfrak{p} \times \mathfrak{p}}$ is a $K$-invariant inner product on $\mathfrak{p}$. Hence $M$ can be equipped with a structure of Riemannian manifold, on which $G$ acts by isometries. The space $M$ is a Riemannian symmetric space of the noncompact type. Up to isomorphism, $M$ does not depend on the choice of $G$, but only on $\mathfrak{g}$ (see [17], Chapter VI). 


\subsection{Hermitian Symmetric Spaces}

The main reference for this section is [17], Chapter VIII). Other relevant sources are [30], [20], Section VII.9 and [14], Sections 1-4 .

Definition 5. A (connected) complex manifold $M$ with a Hermitian metric $h$ is said to be a Hermitian symmetric space if, for each point $m$ in $M$ there exists an involutive holomorphic isometry $s_{m}$ of $M$ such that $m$ is an isolated fixed point of $s_{m}$.

Hermitian symmetric spaces are special cases of Riemannian symmetric spaces (the corresponding Riemannian metric being $g=$ Reh), but we demand that the symmetries be holomorphic. As a consequence, one can show that the group $G$ (the neutral component of the group of $\operatorname{Is}(M, g)$ ) acts by holomorphic transformations on $M$. Notice that $G$ is a real Lie group (and not a complex Lie group).

Using same notation as in previous sections, the tangent space $T_{o} M$ (which is naturally isomorphic to $\mathfrak{p}$ ) admits a complex structure, i.e., there exists a ( $\mathbb{R}$-linear) operator $J=J_{o}$ on $\mathfrak{p}$ which satisfies $J^{2}=-\mathrm{Id}$.

Lemma 6. The complex structure operator J satisfies

$$
\begin{gathered}
J([T, X])=[T, J X] \\
B(J X, J Y)=B(X, Y)
\end{gathered}
$$

for all $X, Y$ in $\mathfrak{p}$ and $T$ in $\mathfrak{k}$.

The first property corresponds to the fact that the action of $K$ on $T_{o} M$ is by complex linear transforms. The second property is a consequence of the fact that $J$ is an isometry of the tangent space for the Riemannian metric $g$.

Lemma 7. Assume that $\mathfrak{g}$ is semisimple. Then there exists a unique element $H$ in the center of $\mathfrak{k}$ such that $J=\operatorname{ad}_{\mathfrak{p}} H$.

Sketch of the proof. The endomorphism $D$ of $\mathfrak{g}$ which is 0 on $\mathfrak{k}$ and coincides with $J$ on $\mathfrak{p}$ is easily seen to be a derivation of $\mathfrak{g}$ (use Lemma 6). If $\mathfrak{g}$ is semisimple, then any derivation is inner, hence $D=\operatorname{ad} H$ for some element $H$ in $\mathfrak{g}$. Decomposing $H$ along $\mathfrak{g}=\mathfrak{k} \oplus \mathfrak{p}$ shows that $H$ has to be in the center of $\mathfrak{k}$.

The fact that $\mathfrak{k}$ has a non trivial center essentially characterizes the Hermitian symmetric spaces among the Riemannian symmetric spaces. In the noncompact type case, a precise statement is the following. 
Proposition 8. Let $\mathfrak{g}$ be a simple Lie algebra of the noncompact type, with Cartan decomposition $\mathfrak{g}=\mathfrak{k} \oplus \mathfrak{p}$. The associated Riemannian symmetric space $M=$ $G / K$ admits a structure of Hermitian symmetric space if and only if the center of $\mathfrak{k}$ is non trivial. If true, there exists a unique (up to \pm 1 ) element $H$ in the center of $\mathfrak{k}$ such that $\mathrm{ad} H$ induces a complex structure operator on $\mathfrak{p}$, and the corresponding symmetric space $M \simeq G / K$ is, in a natural way a Hermitian symmetric space of the noncompact type.

\subsection{The Harish Chandra Embedding}

Proposition 8 gives an abstract description of the Hermitian symmetric space associated to a simple algebra of the noncompact type. A more explicit realization is obtained through the Harish Chandra embedding.

Let $\mathfrak{g}$ be a semi-simple Lie algebra of the noncompact type, with Cartan decomposition $\mathfrak{g}=\mathfrak{k} \oplus \mathfrak{p}$ w.r.t. some Cartan involution $\theta$, and assume that there exists an element $H$ in the center of $\mathfrak{k}$ such that the restriction of $\operatorname{ad} H$ to $\mathfrak{p}$ is a complex structure operator $J$. Let $\mathfrak{G}$ be the complexification of $\mathfrak{g}$ and denote by $X \longmapsto \bar{X}$ the conjugation of $\mathfrak{G}$ with respect to the real form $\mathfrak{g}$. For $Z$ in $\mathfrak{G}$, let $\operatorname{Re} Z=\frac{1}{2}(Z+\bar{Z})$. Extend $\theta$ to $\mathfrak{G}$ in a $\mathbb{C}$-linear way, and observe that $X \mapsto \theta \bar{X}$ is a Cartan involution of $\mathfrak{G}$. Let $\mathfrak{G}=\mathfrak{K} \oplus \mathfrak{P}$ be the complexification of the Cartan decomposition. Extend in a $\mathbb{C}$-linear way the action of $J$ to $\mathfrak{P}$. Then $\mathfrak{P}$ splits as $\mathfrak{P}=\mathfrak{P}_{+} \oplus \mathfrak{P}_{-}$, where $\mathfrak{P}_{ \pm}=\{X \in \mathfrak{P} ; J X= \pm \mathrm{i} X\}$. One can think of $\mathfrak{P}_{+}$as the holomorphic tangent space of $M=G / K$ at the origin $o=e K$.

Lemma 9. The space $\mathfrak{P}_{+}$(respectively $\mathfrak{P}_{-}$) is an Abelian Lie subalgebra of $\mathfrak{G}$. Moreover $\left[\mathfrak{K}, \mathfrak{P}_{ \pm}\right] \subset \mathfrak{P}_{ \pm}$.

Let $\mathbb{G}$ be a complex Lie group with Lie algebra Lie $(\mathbb{G})=\mathfrak{G}$, and define $\mathbb{K}$ (respectively $\mathbb{P}_{+}, \mathbb{P}_{-}$) to be the analytic subgroup of $\mathbb{G}$ with Lie algebra $\mathfrak{K}$ (respectively $\mathfrak{P}_{+}, \mathfrak{P}_{-}$). Let $G$ (respectively $K$ ) be the real analytic subgroup of $\mathbb{G}$ with Lie algebra $\mathfrak{g}$ (respectively $\mathfrak{k}$ ).

Proposition 10. $\quad$ i) The exponential map exp $: \mathfrak{P}_{ \pm} \longrightarrow \mathbb{P}_{ \pm}$is an isomorphism of complex Abelian Lie groups.

ii) $\mathbb{K}$ normalizes $\mathbb{P}_{-}, \mathbb{K} \cap \mathbb{P}_{-}=\{e\}$ and the semidirect product $\mathbb{Q}_{-}=\mathbb{K}_{-}$ is a parabolic subgroup of $\mathbb{G}$.

iii) $\mathbb{P}_{+} \cap \mathbb{Q}_{-}=\{e\}$, and the map

$$
\mathbb{P}_{+} \times \mathbb{Q}_{-} \longrightarrow \mathbb{G}, \quad\left(p_{+}, q\right) \longmapsto p_{+} q
$$


is an injective regular map onto an open subset of $\mathbb{G}$.

iv) $G \subset \mathbb{P}_{+} \mathbb{Q}_{-}$and $G \cap \mathbb{Q}_{-}=K$.

Let $g$ be in $G$. Then, as a consequence of Proposition $10, g$ can be written in a unique way as $g=\exp \Psi(g) q_{-}$, with $q_{-}$in $\mathbb{Q}_{-}$and $\Psi(g)$ in $\mathfrak{P}_{+}$. Moreover, for any $k$ in $K, \Psi(g k)=\Psi(g)$, and hence $\Psi$ induces a map $\zeta: G / K \longrightarrow \mathfrak{P}_{+}$.

Define a norm on $\mathfrak{G}$ by

$$
\|X\|=(-B(X, \theta \bar{X}))^{1 / 2}, \quad X \in \mathfrak{G}
$$

and the corresponding operator norm

$$
\|\operatorname{ad} X\|=\sup \{\|\operatorname{ad} X(Y)\| ; Y \in \mathfrak{G},\|Y\| \leq 1\} .
$$

Theorem 11. (Harish Chandra embedding) The map $\zeta: G / K \longrightarrow \mathfrak{P}_{+}$is a biholomorphic diffeomorphism of $G / K$ onto the domain $\mathcal{D}$, where

$$
\mathcal{D}=\left\{Z \in \mathfrak{P}_{+} ;\|\operatorname{ad}(\operatorname{Re} Z)\|<1\right\}
$$

For a proof, see [17], Chapter VIII and [30], Section II.4.

\subsection{Jordan Triple System}

The rôle of Jordan algebra and Jordan triple system in the theory of Hermitian symmetric spaces is originally due to M. Koecher (see [21]). The notes by O. Loos [25] offer a systematic presentation of the material to be discussed. See also [30] and [14].

Let $M \simeq G / K$ be a Hermitian symmetric space of the noncompact type. Then, considering $M$ has a Riemannian symmetric space, $\mathfrak{p} \simeq T_{o} M$ is equipped with its natural structure of Lie triple system, which coincides (up to a sign) with the curvature tensor at $o$ (see (2)). The behaviour of the curvature tensor under the action of the complex structure $J$ is rather intricate. It leads to the following definition.

For $X, Y, Z$ in $\mathfrak{p}$, let

$$
\{X, Y, Z\}=\frac{1}{2}([[X, Y], Z]+J[[X, J Y], Z])
$$


Theorem 12. The triple product defined by (5) satisfies the following identities

$$
\{X, Y, Z\}=\{Z, Y, X\}
$$

$\{U, V,\{X, Y, Z\}\}=\{\{U, V, X\}, Y, Z\}-\{X,\{V, U, Y\}, Z\}+\{X, Y,\{U, V, Z\}\}$

for all $X, Y, Z, U, V$ in $\mathfrak{p}$.

Moreover it satisfies

$$
[[X, Y], Z]=\{X, Y, Z\}-\{Y, X, Z\}
$$

A complex vector space $\mathbb{V}$ with a triple product $\{X, Y, Z\}$ which is $\mathbb{C}$-linear in $X$ and $Z$, conjugate linear in $Y$, and satisfies JT2) and JT3) is called a Jordan triple system (JTS).

Let $\mathbb{V}$ be a Jordan triple system. For $X$ and $Y$ in $\mathbb{V}$ let $L(X, Y)$ the the $\mathbb{C}$-linear operator on $\mathbb{V}$ defined by

$$
L(X, Y) Z=\{X, Y, Z\}
$$

and consider the sesquilinear form

$$
\tau(X, Y)=\operatorname{Tr} L(X, Y)
$$

If the form $\tau$ is nondegenerate, then $\tau$ is Hermitian (i.e., $\tau(X, Y)=\overline{\tau(Y, X)}$ for $X, Y$ in $\mathbb{V})$. Moreover

$$
L(X, Y)^{*}=L(Y, X)
$$

for $X, Y$ in $\mathbb{V}$, where $A^{*}$ stands for the adjoint of the operator $A$ w.r.t. $\tau$. The triple system is said to be a positive Hermitian Jordan triple system (PHJTS) if the form $\tau$ defined by (7) is positive definite.

Theorem 13. Let $M \simeq G / K$ be a Hermitian symmetric space of the noncompact type. Then $(\mathfrak{p}, J)$ (considered as a complex vector space) with its Jordan triple product defined by (5) is a PHJTS. 


\subsection{An Example: the Hermitian Symmetric Space of Type $I_{p, q}$}

Among the bounded symmetric domains, there the so-called classical ones as opposed to the exceptional ones. The classical ones are studied systematically in [29]. See also [18], and the Appendix of [30]. We present here (to be continued in Sections 3.4 and 4.6) the classical domain of type $I_{p, q}$, i.e., the unit ball in the space of $p \times q$ matrices with complex entries.

Let $\mathbb{E}$ be a complex vector space of dimension $n$, and let $p, q$ two integers, $p, q \geq 1$ with $p+q=n$. Let $\mathbb{G}(q, \mathbb{E})$ be the Grassmannian of all $q$-dimensional vector subspaces of $\mathbb{E}$. It is in a natural way a complex manifold.

Two vector subspaces $\mathbb{W}$ of dimension $q$ and $\mathbb{W}^{\prime}$ of dimension $p$ are said to be transverse if $\mathbb{W} \cap \mathbb{W}^{\prime}=\{0\}$. This relation will be denoted by $\mathbb{W} \top \mathbb{W}^{\prime}$. Choose such a transverse pair $\left(\mathbb{W}_{0}, \mathbb{W}_{0}^{\prime}\right)$ so that $\mathbb{E}=\mathbb{W}_{0}^{\prime} \oplus \mathbb{W}_{0}$. Let

$$
\mathcal{O}_{\mathbb{W}_{0}^{\prime}}:=\left\{\mathbb{W} \in G(q, \mathbb{E}) ; \mathbb{W} \top \mathbb{W}_{0}^{\prime}\right\}
$$

This is an open subset of $\mathbb{G}(q, \mathbb{E})$. Let $L$ be in $\operatorname{Hom}\left(\mathbb{W}_{0}, \mathbb{W}_{0}^{\prime}\right)$, an define its graph $\mathbb{W}_{L}$ as

$$
\mathbb{W}_{L}=\left\{\xi+L \xi ; \xi \in \mathbb{W}_{0}\right\}
$$

Clearly, $\mathbb{W}_{L}$ is a subspace of $\mathbb{E}$ of dimension $q$, and $\mathbb{W}_{L}$ is transverse to $\mathbb{W}_{0}^{\prime}$, hence belongs to $\mathcal{O}_{\mathbb{W}_{0}^{\prime}}$. The map

$$
\operatorname{Hom}\left(\mathbb{W}_{0}, \mathbb{W}_{0}^{\prime}\right) \ni L \longmapsto \mathbb{W}_{L} \in \mathcal{O}_{\mathbb{W}_{0}^{\prime}}
$$

is a chart onto $\mathcal{O}_{\mathbb{W}_{0}^{\prime}}$. The operator $L=0$ corresponds to the "origin" $\mathbb{W}_{0}$ in $\mathcal{O}_{\mathbb{W}_{0}^{\prime}}$. The group $\mathrm{GL}(\mathbb{E}) \simeq \mathrm{GL}(n, \mathbb{C})$ operates transitively on $\mathbb{G}(q, \mathbb{E})$ by $(g, \mathbb{W}) \mapsto$ $g(\mathbb{W})$. Of course, this is really an action of the projective group $\mathbb{G}=\operatorname{PGL}(\mathbb{E}) \simeq$ $\mathrm{GL}(n, \mathbb{C}) / \mathbb{C}^{*}$.

Let $h$ be a Hermitian form on $\mathbb{E}$ of signature $(p, q)$, and let

$$
M=M_{p, q}:=\left\{\mathbb{W} \in \mathbb{G}(q, \mathbb{E}) ; h_{\mid \mathbb{W}} \ll 0\right\} .
$$

Let $\mathbb{W}$ be in $M$. Then the restriction of $h$ to $\mathbb{W}^{\perp}$ is positive-definite and $\mathbb{W}^{\perp}$ is a canonical transverse space to $\mathbb{W}$. It allows to identify the tangent space of $M$ at $\mathbb{W}$ with $\operatorname{Hom}\left(\mathbb{W}, \mathbb{W}^{\perp}\right)$.

Let $G=\mathrm{PU}(\mathbb{E}, h)$ be the (projective) group of (pseudo-)isometries w.r.t $h$. If $g$ is in $G$, and $\mathbb{W}$ is in $M$, then $g(\mathbb{W})$ is still in $M$, so that this defines an action of $G$ on $M$. By Witt's theorem, this action is transitive.

Let $\mathbb{W}$ be in $M$ and $g$ in $\mathrm{U}(\mathbb{E}, h)$. Then $g$ maps $\mathbb{W}$ to $g(\mathbb{W})$, and at the same time, $g\left(\mathbb{W}^{\perp}\right)=(g(\mathbb{W}))^{\perp}$, as $g$ preserves $h$. 
On $M$ there is a natural structure of Hermitian manifold. The tangent space at a point $\mathbb{W}$ has been identified with $\operatorname{Hom}\left(\mathbb{W}, \mathbb{W}^{\perp}\right)$. If $T: \mathbb{W} \rightarrow \mathbb{W}^{\perp}$, define $T^{*}$ to be the unique complex linear operator from $\mathbb{W}^{\perp}$ into $\mathbb{W}$ such that, for all $\xi \in \mathbb{W}$ and $\eta \in \mathbb{W}^{\perp}$.

$$
h(T \xi, \eta)=h\left(\xi, T^{*} \eta\right) .
$$

Then for $T, S$ in $\operatorname{Hom}\left(W, W^{\perp}\right)$ set $h_{\mathbb{W}}(S, T)=\operatorname{Tr} S T^{*}$. This is a positive definite Hermitian form on the tangent space at $\mathbb{W}$, and $h_{\mathbb{W}}$ depends smoothly on $\mathbb{W}$, thus turning $M$ into a Hermitian manifold. Moreover, the stabilizer of $\mathbb{W}$ in $\mathrm{U}(\mathbb{E}, h)$ is $\mathrm{U}\left(\mathbb{W}, h_{\mid \mathbb{W}}\right) \times \mathrm{U}\left(\mathbb{W}^{\perp}, h_{\mid \mathbb{W}} \perp\right.$, and $h_{\mathbb{W}}$ is easily seen to be invariant by this stabilizer. Thus the metric defined by $h_{\mathbb{W}}$ is invariant by $G$.

Fix a point $\mathbb{W}_{0}$ in $M$. Then $\mathbb{E}=\mathbb{W}_{0} \oplus \mathbb{W}_{0}^{\perp}$, and let $\sigma_{0}=\sigma_{\mathbb{W}_{0}}$ be the symmetry with respect to this decomposition, defined by $\sigma_{0}(\xi+\eta)=\xi-\eta$ for $\xi \in \mathbb{W}_{0}$ and $\eta \in \mathbb{W}_{0}^{\perp}$. This symmetry belongs to $U(\mathbb{E}, h)$ and hence operates on $M$. Let $\mathbb{W}$ in $M$ be a fixed point of $\sigma_{0}$. Thus $\mathbb{W}$ is stable by $\sigma_{0}$, hence decomposes as $\mathbb{W}=\mathbb{W} \cap \mathbb{W}_{0} \oplus \mathbb{W} \cap \mathbb{W}_{0}^{\perp}$. As the restriction of $h$ to $\mathbb{W}$ has to be negative definite, this forces $\mathbb{W}=\mathbb{W}_{0}$. Thus $\sigma_{0}$ acts on $M$ by an involutive holomorphic transformation, preserving the Hermitian metric and having $\mathbb{W}_{0}$ as isolated (even unique) fixed point. This shows that $M$ is a Hermitian symmetric space.

The Lie algebra of $\mathrm{PU}(\mathbb{E}, h)$ is the same as the Lie algebra of $\mathrm{SU}(\mathbb{E}, h)$ as both groups are locally isomorphic, and it is given by

$\mathfrak{g}=\mathfrak{s u}(\mathbb{E}, h)=\{X \in \operatorname{End}(\mathbb{E}) ; h(X \xi, \eta)+h(\xi, X \eta)=0, \xi, \eta \in \mathbb{E}, \operatorname{Tr} X=0\}$.

Choose a basis $\left(e_{1}, \ldots, e_{p}, e_{p+1}, \ldots, e_{n}\right)$, of $\mathbb{E}$ such that $h\left(e_{i}, e_{i}\right)=1$ for $1 \leq$ $i \leq p, h\left(e_{i}, e_{i}\right)=-1$ for $p+1 \leq i \leq n$ and $h\left(e_{i}, e_{j}\right)=0$ for $1 \leq i \neq j \leq n$, so that the form $h$ is represented by the matrix

$$
I_{p, q}=\left(\begin{array}{cccccc}
1 & & & & & \\
& \ddots & & & & \\
& & 1 & & & \\
& & & -1 & & \\
& & & & \ddots & \\
& & & & & -1
\end{array}\right)
$$

The Lie algebra $\mathfrak{g}$ of $G$ is then given by

$$
\mathfrak{g}=\mathfrak{s u}(p, q)=\left\{X=\left(\begin{array}{cc}
A & Y \\
Y^{*} & D
\end{array}\right) ; A^{*}=-A, D^{*}=-D, \quad \operatorname{Tr}(A+D)=0\right\}
$$


The Lie algebra $\mathfrak{k}$ of $K \simeq \mathrm{P}(\mathrm{U}(q) \times \mathrm{U}(p))$ is

$$
\mathfrak{k}=\mathfrak{s}(\mathfrak{u}(p) \times \mathfrak{u}(q))=\left\{X=\left(\begin{array}{cc}
A & 0 \\
0 & D
\end{array}\right), A=-A^{*}, D^{*}=-D, \operatorname{Tr} A+\operatorname{Tr} D=0\right\}
$$

whereas $\mathfrak{p}$ is given by

$$
\mathfrak{p}=\left\{X=\left(\begin{array}{cc}
0 & Y \\
Y^{*} & 0
\end{array}\right) ; Y \in \operatorname{Mat}(p \times q, \mathbb{C})\right\}
$$

which allows to identify $\mathfrak{p}$ with $\operatorname{Mat}(p \times q, \mathbb{C})$.

The element $H$ which is in the center of $\mathfrak{k}$ such that $\operatorname{ad}_{\mathfrak{p}} H$ is the complex structure operator on $\mathfrak{p}$ is

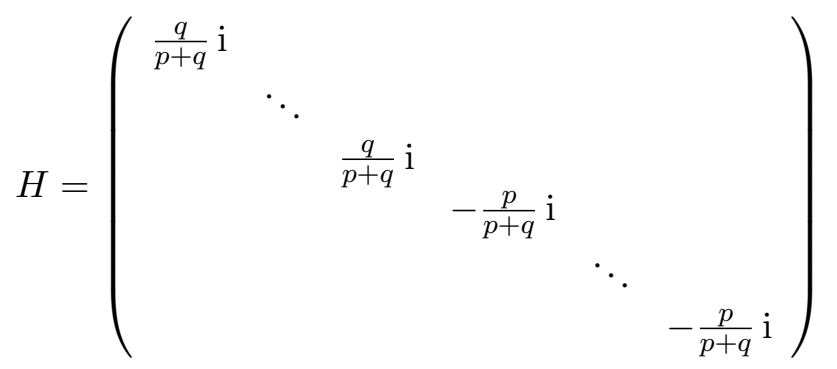

The Lie triple system on $\mathfrak{p}$ is given by

$$
[X, Y, Z]=[[X, Y], Z]=X Y^{*} Z-Y X^{*} Z-Z X^{*} Y+Z Y^{*} X
$$

for $X, Y, Z p \times q$ matrices.

The complexified Lie algebra $\mathfrak{G}$ of $\mathfrak{g}$ can be realized as $\mathfrak{s l}(n, \mathbb{C})$, the Lie algebra of $n \times n$ matrices with trace 0 . Then

$$
\mathfrak{K}=\left\{\left(\begin{array}{cc}
A & 0 \\
0 & B
\end{array}\right) ; \operatorname{Tr} A+\operatorname{Tr} B=0\right\}, \quad \mathfrak{P}=\left\{\left(\begin{array}{cc}
0 & Y \\
Z & 0
\end{array}\right)\right\}
$$

and

$$
\mathfrak{P}^{+}=\left\{\left(\begin{array}{cc}
0 & Y \\
0 & 0
\end{array}\right)\right\}
$$

The corresponding Jordan triple product is given by

$$
\{X, Y, Z\}=X Y^{*} Z+Z Y^{*} X \text {. }
$$




\section{Bounded Symmetric Domains}

\subsection{Bergman Metric}

The reference for this section is [17], Chapter VIII.

Let $\mathcal{D}$ be a domain (i.e., a open connected subset) in some complex finite-dimensional vector space $\mathbb{E}$. Choose a Lebesgue measure $\mathrm{d} \lambda$ on $\mathbb{E}$.

Definition 14. Let

$$
\mathcal{H}(\mathcal{D})=\left\{f: \mathcal{D} \longrightarrow \mathbb{C} ; f \text { holomorphic, } \int_{\mathcal{D}}|f(z)|^{2} \mathrm{~d} \lambda(z)<\infty\right\}
$$

The Bergman space $\mathcal{H}(\mathcal{D})$ is the Hilbert space equiped with the norm $\|f\|=$ $\left(\int_{\mathcal{D}}|f(z)|^{2} \mathrm{~d} \lambda(z)\right)^{1 / 2}$, as it is a closed subspace of $L^{2}(\mathcal{D})$. Let $w$ be in $\mathcal{D}$. Then the linear functional

$$
\mathcal{H}(\mathcal{D}) \ni f \longmapsto f(w)
$$

is continuous (the proof uses the Cauchy formula and Schwarz inequality). Hence there exists $K_{w}$ in $\mathcal{H}(\mathcal{D})$ such that

$$
f(w)=\int_{\mathcal{D}} f(z) \overline{K_{w}(z)} \mathrm{d} \lambda(z)=\int_{\mathcal{D}} f(z) \overline{k(z, w)} \mathrm{d} \lambda(z)
$$

where $k(z, w)=K_{w}(z)$ is called the Bergman kernel of the domain $\mathcal{D}$.

Proposition 15. The Bergman kernel satisfies the following properties:

i) $k(z, w)$ is holomorphic in $z$ and conjugate holomorphic in $w$.

ii) $k(z, w)=\overline{k(w, z)}$

iii) for any biholomorphic diffeomorphism $\Phi$ of $\mathcal{D}$

$$
k(z, w)=j_{\Phi}(z) k(\Phi(z), \Phi(w)) \overline{j_{\Phi}(w)}
$$

where $j_{\Phi}(z)$ is the Jacobian of $\Phi$ at $z$.

Assume that $\mathcal{D}$ is a bounded domain. Then $\mathcal{H}(\mathcal{D})$ is not equal to $\{0\}$, as it contains the restrictions to $\mathcal{D}$ of all holomorphic polynomials on $\mathbb{E}$. As a consequence, for 
any $z$ in $\mathcal{D}, k(z, z)>0$, and the Bergman kernel can be used to construct a Hermitian metric on $\mathcal{D}$ given by

$$
h_{z}(\xi, \eta)=\partial_{\xi} \overline{\partial_{\eta}} \log k(u, w)_{u=z, w=z}
$$

called the Bergman metric. The Bergman metric is invariant under any holomorphic diffeomorphism of $\mathcal{D}$ (a consequence of (10)).

Remark. Suppose $\mathcal{D}$ is a homogeneous domain, i.e., its group of biholomorphic diffeomorphisms $G$ is transitive on $\mathcal{D}$. Fix an origin $o$ in $\mathcal{D}$. Then, by (10), $k(z, z)=k(o, o)\left|j_{g}(z)\right|^{-2}$, where $g$ is any element of $G$ such that $z=g(o)$. Now $k(z, w)$, being holomorphic in $z$ and conjugate holomorphic in $w$ is determined by its restriction to the diagonal. In practice, this gives, for a homogeneous bounded domain, a way of computing explicitely the Bergman kernel (up to a positive constant) and the corresponding Bergman metric.

\subsection{Bounded Symmetric Domains}

In this section we mainly follow [25], Section 2.

Definition 16. A bounded domain $\mathcal{D}$ is said to be symmetric ( $\mathcal{D}$ is also called a Cartan domain) if, for every $z$ in $\mathcal{D}$, there exists an involutive biholomorphic diffeomorphism $s_{z}$ such that $z$ is an isolated fixed point of $s_{z}$.

The use of Bergman metric of $\mathcal{D}$ implies that $\mathcal{D}$ is a Hermitian symmetric space of the noncompact type. Let $G$ be the neutral component of the group of holomorphic diffeomorphisms of $\mathcal{D}$, and let $K$ be the stabilizer in $G$ of some fixed origin $o$ in $\mathcal{D}$. Then $G$ is a semisimple Lie group, $K$ is a maximal compact subgroup and $\mathcal{D}$ is isomorphic to $G / K$.

A domain $\mathcal{D}$ is said to be circled if 0 belongs to $\mathcal{D}$ and $\mathcal{D}$ is stable by the maps $r_{\theta}: z \longmapsto \mathrm{e}^{\mathrm{i} \theta} z$, for $\theta \in \mathbb{R} / 2 \pi \mathbb{Z}$.

Theorem 17. A bounded symmetric domain is holomorphically equivalent to a bounded symmetric and circled domain.

See [33] for a proof (the result is valid even in infinite dimension).

Let $\mathcal{D}$ be a bounded circled symmetric domain. Choose 0 as origin in $\mathcal{D}$. Then the stabilizer $K$ of 0 in $G$ acts by (restrictions to $\mathcal{D}$ of) linear transformations. The Hermitian form $h_{0}$ on $T_{0} \mathcal{D} \simeq \mathbb{E}$ given by the Bergman metric at 0 is invariant 
under $K$, so that $K$ can be viewed as a closed subgroup of the unitary group $\mathrm{U}\left(\mathbb{E}, h_{0}\right)$. The symmetry $s_{0}$ is given by

$$
s_{0}: z \mapsto-z=\mathrm{e}^{\mathrm{i} \pi} z
$$

and belongs to $K$, as $\mathcal{D}$ is circled. The map $\theta: g \longmapsto s_{0} \circ g \circ s_{0}$ is a Cartan involution of $G$, with $K$ as fixed points.

Let $\mathfrak{g}=\mathfrak{k} \oplus \mathfrak{p}$ be the Cartan decomposition of the Lie algebra $\mathfrak{g}$ of $G$ with respect to $\theta$.

A holomorphic vector field on $\mathcal{D}$ can be regarded as a holomorphic map $\xi_{X}$ : $\mathcal{D} \longrightarrow \mathbb{E}$. In this setting, the bracket of two holomorphic vector fields $\xi$ and $\eta$ is the holomorphic vector field $[\xi, \eta]$ defined by

$$
[\xi, \eta](z)=D \eta(z) \xi(z)-D \xi(z) \eta(z) .
$$

Any $X$ in $\mathfrak{g}$ induces a holomorphic vector field in $\mathcal{D}$ denoted by $\xi_{X}$. The map $X \longmapsto \xi_{X}(0)$ yields a real isomorphism of $\mathfrak{p}$ with $\mathbb{E}$, which is $K$-equivariant.

For $X$ and $Y$ in $\mathfrak{g}$, one has the relation

$$
\xi_{[X, Y]}=-\left[\xi_{X}, \xi_{Y}\right] .
$$

For $u$ in $\mathbb{E}$, abusing notation, denote by $\xi_{u}$ the unique holomorphic vector field induced by an element of $\mathfrak{p}$ such that $\xi_{u}(0)=u$.

Proposition 18. Let $v$ be in $\mathbb{E}$. Then, for any $z$ in $\mathcal{D}$

$$
\xi_{v}(z)=v-Q(z) v
$$

where $Q(z)$ is a conjugate linear map of $\mathbb{E}$, and $z \mapsto Q(z)$ is a homogeneous quadratic map of degree two.

For $u, v$ in $\mathbb{E}$, set

$$
Q(u, v)=Q(u+v)-Q(u)-Q(v)
$$

(polarized symmetric form of $Q$, except for a factor two), and for $x, y, z$ in $\mathbb{E}$

$$
\{x, y, z\}=Q(x, z) y
$$

Theorem 19. The formula (16) defines on $\mathbb{E}$ a structure of positive Hermitian Jordan triple system (PHJTS) which coincides with the structure on $\mathfrak{p}$ defined by (5). 


\subsection{The Spectral Norm on $\mathbb{E}$}

The main reference for this section is [25], Section 3 and 5. Let $\mathbb{E}$ be a PHJTS.

Definition 20. A real subspace $W$ of $\mathbb{E}$ is said to be flat if

$$
\begin{gathered}
\{W, W, W\} \subset W \\
\text { for any } x, y \in W, \quad\{x, y, z\}=\{y, x, z\} .
\end{gathered}
$$

Let $W$ be a flat subspace of $\mathbb{E}$. For $x, y$ in $W$, denote by $\widetilde{L}(x, y)$ the restriction to $W$ of $L(x, y)$. For $x, y, u$ arbitrary elements of $\mathbb{E}$, rewrite JT3) as

$$
[L(x, y), L(u, u)]=L(\{x, y, u\}, u)-L(u,\{y, x, u\}) .
$$

Now, if $x, y, u$ are in $W$, then, by (17) and (18), $\widetilde{L}(\{x, y, u\}, u)=\widetilde{L}(u,\{x, y, u\})=$ $\widetilde{L}(u,\{y, x, u\})$, so that $\widetilde{L}(x, y)$ commutes to $\widetilde{L}(u, u)$, and hence, by polarization, to $\widetilde{L}(u, v)$ for arbitrary $u$ and $v$ in $W$.

The restriction to $W$ of the real part of the Hermitian form $\tau$ is a real inner product on $W$, and as a consequence of $(8), \widetilde{L}(x, y)^{t}=\widetilde{L}(y, x)=\widetilde{L}(x, y)$.

Hence the family $\{\widetilde{L}(x, y), x, y \in W\}$ is a family of mutually commuting symmetric operators on $W$, so that there is a simultaneous diagonalization of the family. This result allows a spectral analysis in $\mathbb{E}$.

An element $c$ of $\mathbb{E}$ is said to be a tripotent if it satisfies

$$
\{c, c, c\}=2 c .
$$

Two tripotents $c$ and $d$ are said to be orthogonal if $L(c, d)=0$. If this is the case, then $c+d$ is a tripotent.

Let $c$ be a tripotent. One can show that $L(c, c)$ (which is selfadjoint) admits eigenvalues in the set $\{2,1,0\}$. There is a corresponding decomposition of $\mathbb{E}$, called the Peirce decomposition with respect to $c$

$$
\mathbb{E}=\mathbb{E}_{2}(c) \oplus \mathbb{E}_{1}(c) \oplus \mathbb{E}_{0}(c)
$$

where $\mathbb{E}_{j}(c)=\{x \in \mathbb{E} ; L(c, c) x=j x\}$, for $j=0,1,2$.

Proposition 21. Let c be a tripotent in $\mathbb{E}$. Then the Peirce decomposition (20) has the following properties:

$$
\begin{gathered}
\left\{\mathbb{E}_{i}(c), \mathbb{E}_{j}(c), \mathbb{E}_{k}(c)\right\} \subset \mathbb{E}_{i-j+k}(c) \\
\left\{\mathbb{E}_{2}(c), \mathbb{E}_{0}(c), \mathbb{E}\right\}=0, \quad\left\{\mathbb{E}_{0}(c), \mathbb{E}_{2}(c), \mathbb{E}\right\}=0
\end{gathered}
$$

where $i, j, k$ belong to $\{0,1,2\}$ and with the convention that $\mathbb{E}_{l}(c)=\{0\}$ if $l$ does not belong to $\{0,1,2\}$. 
Theorem 22. Let $c_{1}, c_{2}, \ldots, c_{s}$ be a family of mutually orthogonal nonzero tripotents. Then

$$
W=\mathbb{R} c_{1} \oplus \mathbb{R} c_{2} \oplus \cdots \oplus \mathbb{R} c_{s}
$$

is a flat subspace of $\mathbb{E}$. Conversely, let $W$ be a flat subspace of $W$. Then there exists a family $\left(c_{1}, c_{2}, \ldots, c_{s}\right)$ of mutually orthogonal tripotents such that (21) yields. Moreover, the family is unique, up to order and signs.

If $x$ is any element of $\mathbb{E}$, its odd powers are defined by the induction formula

$$
x^{(2 p+1)}=\left\{x, x^{(2 p-1)}, x\right\} .
$$

The real vector space $\mathbb{R}[x]$ generated by the odd powers of $x$ is a flat subspace. The previous result implies the following spectral theorem.

Theorem 23. Let $x$ be an element of $\mathbb{E}$. Then there exists a unique family of mutually orthogonal tripotents $c_{1}, c_{2}, \ldots, c_{s}$, and such positive real numbers $0<$ $\lambda_{1}<\lambda_{2}<\cdots<\lambda_{s}$ that

$$
x=\lambda_{1} c_{1}+\lambda_{2} c_{2}+\cdots+\lambda_{s} c_{s} .
$$

The $\lambda_{j}$ 's are called the spectral values of $x$. The spectral norm is, by definition the largest eigenvalue of $x$ and is denoted by $|x|$. It can be shown that $x \longmapsto|x|$ is actually a (complex Banach) norm on $\mathbb{E}$.

Theorem 24. Let $\mathcal{D}$ be a bounded circled domain in some complex vector space $\mathbb{E}$. Let $\{., .,$.$\} be the induced structure of PHJTS on \mathbb{E}$, and let $|$.$| be the corre-$ sponding spectral norm. Then

$$
\mathcal{D}=\{x \in \mathbb{E} ;|x|<1\} .
$$

Conversely, let $\mathbb{E}$ be a PHJTS. The open unit ball for the spectral norm is a bounded symmetric domain.

\subsection{An Example (Continued from Section 2.5)}

We continue to use notation introduced in Section $\mathbf{2 . 5}$ for the Hermitian symmetric space $M_{p, q}$. Recall that we chose an orthogonal decomposition $\mathbb{E}=\mathbb{W}_{0}^{\perp} \oplus \mathbb{W}_{0}$, with $h_{\mid \mathbb{W}_{0} \perp} \gg 0$ and $h_{\mid \mathbb{W}_{0}} \ll 0$. The restriction of $h$ to $\mathbb{W}_{0}{ }^{\perp}$ yields an inner product on $\mathbb{W}_{0}{ }^{\perp}$, and similarly for the restriction of $(-h)$ to $\mathbb{W}_{0}$. We denote 
the corresponding Hilbert norm on both of them by $\|$.$\| , and we also define the$ corresponding operator norm on $\operatorname{Hom}\left(\mathbb{W}_{0}, \mathbb{W}_{0}{ }^{\perp}\right)$ by

$$
\|Z\|=\sup \left\{\|Z \xi\| ; \xi \in \mathbb{W}_{0},\|\xi\| \leq 1\right\} .
$$

Let $\mathbb{W}$ be in $M$, so that $h_{\mid \mathbb{W}} \ll 0$. Then $\mathbb{W} \cap \mathbb{W}_{0} \perp=\{0\}$, and $\mathbb{W}$ belongs to $\mathcal{O}_{\mathbb{W}_{0}} \perp$. Hence there is a map $Z: \mathbb{W}_{0} \rightarrow \mathbb{W}_{0}^{\perp}$ such that

$$
\mathbb{W}=\mathbb{W}_{Z}:=\left\{\xi+Z \xi ; \xi \in \mathbb{W}_{0}\right\}
$$

Conversely, let $Z$ be in $\operatorname{Hom}\left(\mathbb{W}_{0}, \mathbb{W}_{0}^{\perp}\right)$ and let $\mathbb{W}_{Z}$ be its graph. Then the condition $h_{\mid \mathbb{W}_{Z}} \ll 0$ reads

$$
h(Z \xi, Z \xi)<-h(\xi, \xi), \quad \text { for any } \xi \neq 0 \in \mathbb{W}_{0}
$$

which is equivalent to the condition $\|Z\|<1$.

Denote by $\mathcal{D}_{p, q}$ the unit ball in $\operatorname{Hom}\left(\mathbb{W}_{0}, \mathbb{W}_{0}{ }^{\perp}\right) \simeq \operatorname{Mat}(p \times q, \mathbb{C})$ for the operator norm. We just proved the following result, which describes the Harish Chandra embedding for the Hermitian symmetric space $M_{p, q}$.

Proposition 25. The map $\quad Z \longmapsto \mathbb{W}_{Z} \quad$ is a 1-1 correspondance between $\mathcal{D}_{p, q}$ and $M_{p, q}$.

Next we want to make explicit the action of $G=\mathrm{PU}(p, q)$ on $\mathcal{D}_{p, q}$. Let $g$ be in $\mathrm{U}(p, q)$. Its block matrix expression with respect to the decomposition of $\mathbb{E}$ as $\mathbb{E}=\mathbb{W}_{0} \perp \oplus \mathbb{W}_{0}$ is of the form

$$
g=\left(\begin{array}{ll}
a & b \\
c & d
\end{array}\right), \quad\left\{\begin{array}{l}
a^{*} a-c^{*} c=1_{p} \\
b^{*} a-d^{*} c=0 \\
b^{*} b-d^{*} d=-1_{q} .
\end{array}\right.
$$

Let $Z$ be in $\operatorname{Hom}\left(\mathbb{W}_{0}, \mathbb{W}_{0}^{\perp}\right)$. Then for any $\xi$ in $\mathbb{W}_{0}$

$$
g\left(\begin{array}{c}
Z \xi \\
\xi
\end{array}\right)=\left(\begin{array}{c}
(a Z+b) \xi \\
(c Z+d) \xi
\end{array}\right)
$$

If $\|Z\|<1$, then $c Z+d$ is invertible, and letting $\eta=(c Z+d) \xi$, we obtain that $g\left(\mathbb{W}_{Z}\right)=\mathbb{W}_{g(Z)}$ where

$$
g(Z)=(a Z+b)(c Z+d)^{-1}
$$

Now let $Y$ be in $\mathfrak{p} \simeq \operatorname{Hom}\left(\mathbb{W}_{0}, \mathbb{W}_{0}{ }^{\perp}\right)$. Then, for $t$ in $\mathbb{R}$ close to 0

$$
\exp t Y=\left(\begin{array}{cc}
1_{p} & t Y \\
t Y^{*} & 1_{q}
\end{array}\right)+O\left(t^{2}\right)
$$


and hence

$\exp (t Y)(Z)=(Z+t Y)\left(t Y^{*} Z+1_{q}\right)^{-1}+0\left(t^{2}\right)=Z+t\left(Y-Z Y^{*} Z\right)+O\left(t^{2}\right)$

so that the holomorphic vector field induced by $Y$ is given by

$$
\xi_{Y}(Z)=Y-Z Y^{*} Z
$$

Hence the Jordan triple product on $\mathbb{V}=\operatorname{Hom}\left(\mathbb{W}_{0}, \mathbb{W}_{0}^{\perp}\right)$ reads

$$
Q(Z) Y=Z Y^{*} Z, \quad\{X, Y, Z\}=X Y^{*} Z+Z Y^{*} X .
$$

Tripotents for this Jordan triple system are obtained as follows. Let $\mathbb{F}^{\prime} \subset \mathbb{W}_{0}{ }^{\perp}$ and $\mathbb{F} \subset \mathbb{W}_{0}$ be two subspaces of the same dimension, say $s$ with $0 \leq s \leq r=$ $\inf (p, q)$. Denote by $\mathbb{F}^{\perp}$ (respectively $\mathbb{F}^{\prime}$ ) the orthogonal of $\mathbb{F}$ (respectively $\mathbb{F}^{\prime}$ ) in $\mathbb{W}_{0}$ (respectively $\mathbb{W}_{0}^{\perp}$ ). Let $c: \mathbb{F} \longrightarrow \mathbb{F}^{\prime}$ be an isometry. Associate to $c$ the map $C: \mathbb{W}_{0} \longrightarrow \mathbb{W}_{0}{ }^{\perp}$ as the following composed map

$$
C: \mathbb{W}_{0} \stackrel{p}{\rightarrow} \mathbb{F} \stackrel{c}{\rightarrow} \mathbb{F}^{\prime} \stackrel{i}{\rightarrow} \mathbb{W}_{0} \perp
$$

where $p$ is the orthogonal projection on $\mathbb{F}$ and $i$ is the canonical injection in $\mathbb{W}_{0}{ }^{\perp}$. Then $C$ is a tripotent, and any tripotent is obtained in this manner. Observe that $\mathbb{F}=(\operatorname{ker} C)^{\perp}$ and $\mathbb{F}^{\prime}=\operatorname{im} C$.

The Peirce decomposition $\mathbb{V}=\mathbb{V}_{2}(C) \oplus \mathbb{V}_{1}(C) \oplus \mathbb{V}_{0}(C)$ w.r.t. $C$ is described in terms of block matrices w.r.t. the orthogonal decompositions $\mathbb{W}=\mathbb{F} \oplus \mathbb{F}^{\perp}$ and $\mathbb{W}_{0}{ }^{\perp}=\mathbb{F}^{\prime} \oplus \mathbb{F}^{\prime} \perp$ by the following symbolic scheme

$$
\left.\begin{array}{lcc} 
& \mathbb{F} & \mathbb{F}^{\perp} \\
\mathbb{F}^{\prime} & \left(\mathbb{V}_{2}(C)\right. & \mathbb{V}_{1}(C) \\
\mathbb{F}^{\prime} \perp & \left(\mathbb{V}_{1}(C)\right. & \mathbb{V}_{0}(C)
\end{array}\right) .
$$

Two tripotents $C_{1}, C_{2}$ associated to the subspaces $\left(\mathbb{F}_{1}, \mathbb{F}_{1}^{\prime}\right)$ and $\left(\mathbb{F}_{2}, \mathbb{F}_{2}^{\prime}\right)$ respectively are orthogonal if and only if $\mathbb{F}_{1} \perp \mathbb{F}_{2}$ and $\mathbb{F}_{1}^{\prime} \perp \mathbb{F}_{2}^{\prime}$.

Let $X$ be any element in $\operatorname{Hom}\left(\mathbb{W}_{0}, \mathbb{W}_{0}{ }^{\perp}\right)$. Let $\mathbb{F}=(\operatorname{ker} X)^{\perp}$ and $\mathbb{F}^{\prime}=\operatorname{im} X$. On $\mathbb{F}$, consider the Hermitian form defined by

$$
q_{X}\left(\xi, \xi^{\prime}\right)=h\left(X \xi, X \xi^{\prime}\right)
$$

which is a positive definite form on $\mathbb{F}$, and can be compared to the positive definite form $-h_{\mid \mathbb{F}}$. Hence there exists an orthonormal basis $\left(\xi_{1}, \xi_{2}, \ldots, \xi_{s}\right)$ of $\mathbb{F}$ such that the matrix of the form $q_{X}$ in this basis is diagonal with positive entries (say) $\lambda_{1}^{2}, \lambda_{2}^{2}, \ldots, \lambda_{s}^{2}$, where we assume that $0<\lambda_{1} \leq \lambda_{2} \leq \cdots \leq \lambda_{s}$. For $1 \leq$ 
$j \leq s$, let $\eta_{j}=\frac{X \xi_{j}}{\lambda_{j}}$. Then $\eta_{1}, \eta_{2}, \ldots, \eta_{s}$ is an orthonormal basis of $\mathbb{F}^{\prime}$. For $1 \leq j \leq s$, let $C_{j}$ be the tripotent (of rank one) associated to the isometry $c_{j}$ : $\mathbb{C} \xi_{j} \rightarrow \mathbb{C} \eta_{j}$ which maps $\xi_{j}$ to $\eta_{j}$. Then the $C_{j}$ are orthogonal tripotents, and $X=\sum_{j=1}^{s} \lambda_{j} C_{j}$. This is essentially the spectral decomposition of $X$ in the sense of Theorem 23. The $\lambda_{j}$ 's are usually called the singular spectral values of $X$. The largest eigenvalue $\lambda_{\max }$ is given

$$
\begin{aligned}
\lambda_{\max }^{2} & =\sup \left\{q_{X}(\xi, \xi) ; \xi \in \mathbb{F},\|\xi\| \leq 1\right\} \\
& =\sup \left\{\|X \xi\|^{2} ; \xi \in \mathbb{W}_{0},\|\xi\| \leq 1\right\}=\|X\|_{o p}^{2}
\end{aligned}
$$

so that the spectral norm on the PHJTS Hom $\left(\mathbb{W}_{0}, \mathbb{W}_{0}{ }^{\perp}\right)$ coincides with the operator norm.

The Bergman kernel of the domain $\mathcal{D}$ is given by

$$
k(Z, W)=c_{p, q} \operatorname{det}\left(1_{p}-Z W^{*}\right)^{-n} .
$$

with $c_{p, q}$ a positive real number (see [18]).

\section{The Shilov Boundary of a Bounded Symmetric Domain}

The presentation of this section follows [25], Section 6.

\subsection{The Shilov Boundary of a Bounded Domain}

Let $\mathcal{D}$ be a bounded domain in some complex finite-dimensional vector space $\mathbb{V}$. Let $f$ be a function defined and holomorphic in a neighbourhood of $\overline{\mathcal{D}}$. Then the classical maximum principle asserts that $\sup _{z \in \overline{\mathcal{D}}}|f(z)|$ is reached on the boundary $\partial \mathcal{D}$ of $\mathcal{D}$. It is a typical phenomenon of the theory of holomorphic functions in several complex variables that this result may not be optimal. A closed subset $F$ of $\partial \mathcal{D}$ is said to satisfy the maximum principle for holomorphic functions if, for any function $f$ defined and holomorphic in a neighbourhood of $\overline{\mathcal{D}}$

$$
\sup _{z \in \overline{\mathcal{D}}}|f(z)|=\sup _{z \in F}|f(z)|
$$

in other words if the maximum of $|f|$ over $\overline{\mathcal{D}}$ is reached in $F$. One can show that there exists a (unique) smallest closed set which satisfies the maximum principle, called the Shilov boundary of the bounded domain $\mathcal{D}$. 
Example 1. Let $\mathcal{D}=\left\{\left(z_{1}, z_{2}\right) \in \mathbb{C}^{2} ;\left|z_{1}\right|<1,\left|z_{2}\right|<1\right\}$ be the product of two copies of the complex unit disc. Its topological boundary is

$$
\partial D=\left\{\left(z_{1}, z_{2}\right) \in \mathbb{C}^{2} ;\left|z_{1}\right|,\left|z_{2}\right| \leq 1,\left|z_{1}\right|=1 \text { or }\left|z_{2}\right|=1\right\}
$$

whereas its Shilov boundary is

$$
S=\left\{\left(z_{1}, z_{2}\right) \in \mathbb{C}^{2} ;\left|z_{1}\right|=\left|z_{2}\right|=1\right\} .
$$

The last statement is obtained by appplying twice the maximum principle with respect to each complex variable.

Example 2. Let $\mathcal{D}=\mathcal{D}_{p, q}$ be the unit ball (for the standard operator norm) in $\operatorname{Mat}(p \times q, \mathbb{C})$, and assume $p \geq q$. A matrix $A$ is in the topological boundary of $\mathcal{D}$ if and only if $\|A\|_{o p}=1$. Equivalently, $A^{*} A$ has all its eigenvalues less than or equal to 1 , and 1 is an eigenvalue of $A^{*} A$. Now $A$ is in the Shilov boundary of $\mathcal{D}$ if and only if $A^{*} A=\mathrm{id}_{q}$ (see Section $\mathbf{4 . 6}$ for a proof of this result). The topological boundary and the Shilov boundary of $\mathcal{D}$ coincide if and only if $q=1$, i.e., if the domain $\mathcal{D}$ is the unit ball in $\mathbb{C}^{p}$.

Next, we will characterize the Shilov boundary of the unit ball of a PHJTS.

\subsection{More on Tripotents}

Let $\mathbb{V}$ be a PHJTS, and let $\mathcal{D}$ be its unit ball for the spectral norm. More generally, we use freely of the notation introduced in Sections $\mathbf{2}$ and $\mathbf{3}$. Recall that a tripotent is an element $c$ of $\mathbb{V}$ which satisfies $\{c, c, c\}=2 c$.

There is a partial order on tripotents: if $c$ and $d$ are two tripotents, then say that $c \prec d$ if there exists a tripotent $f \neq 0$ orthogonal to $c$ and such that $d=c+f$. A nonzero tripotent $c$ is said to be primitive if it can not be written as a sum of two nonzero orthogonal tripotents. In other words, a primitive tripotent is a minimal element among the nonzero tripotents.

A Peirce frame is, by definition, a maximal set of mutually orthogonal primitive tripotents.

Proposition 26. Let $c$ be a tripotent of $\mathbb{V}$. Then the following are equivalent:

i) $c=c_{1}+c_{2}+\cdots+c_{r}$, where $\left(c_{1}, c_{2}, \ldots, c_{r}\right)$ is a Peirce frame.

ii) $c$ is a maximal tripotent

iii) in the Peirce decomposition of $\mathbb{V}$ with respect to $c$, the factor $\mathbb{V}_{0}(c)$ is equal to $\{0\}$ and hence $\mathbb{V}=\mathbb{V}_{2}(c) \oplus \mathbb{V}_{1}(c)$. 
In this context, the spectral theorem (cf Th. 23) can be written slightly differently.

Theorem 27. Let $x$ be an element in $\mathbb{V}$. Then there exists such a Peirce frame $\left(c_{1}, c_{2}, \ldots, c_{r}\right)$ and nonnegative real numbers $0 \leq \lambda_{1} \leq \cdots \leq \lambda_{r}$ that $x=$ $\sum_{j=1}^{r} \lambda_{j} c_{j}$.

Let $\mathbb{V}_{1}$ and $\mathbb{V}_{2}$ be two PHJTS. Then the direct sum $\mathbb{V}=\mathbb{V}_{1} \oplus \mathbb{V}_{2}$ has a natural structure of PHJTS, simply by setting

$$
\left\{x_{1}+x_{2}, y_{1}+y_{2}, z_{1}+z_{2}\right\}=\left\{x_{1}, y_{1}, z_{1}\right\}_{1}+\left\{x_{2}, y_{2}, z_{2}\right\}_{2} \text {. }
$$

The spectral norm on $\mathbb{V}$ is given

$$
\left|\left(x_{1}+x_{2}\right)\right|=\sup \left(\left|x_{1}\right|_{1},\left|x_{2}\right|_{2}\right)
$$

A PHJTS $\mathbb{V}$ is said to be simple if it can not be written as a sum of two PHJTS. The simplicity of $\mathbb{V}$ is equivalent to the fact that the unit ball $\mathcal{D}$ is irreducible as Hermitian symmetric space.

Proposition 28. Let $\mathbb{V}$ be a PHJTS. Then

i) two Peirce frames are conjugate under $K$.

ii) two maximal tripotents are conjugate under $K$.

iii) Assume that $\mathbb{V}$ is a simple PHJTS. Then two minimal tripotents are conjugate under $K$.

From now on, we will assume, mostly for convenience, that $\mathbb{V}$ is a simple PHJTS, although many statements are true generally or could be reformulated to be valid in full generality.

The number of elements of a Peirce frame is the same for all frames, and is called the rank of $\mathbb{V}$, denoted by $r$. It is the rank of a maximal tripotent. It is also equal to the rank of $\mathcal{D}$ as Hermitian symmetric space.

Let $c$ be a tripotent. Then $c$ can be written as a sum of primitive tripotents, and the number of tripotents is the same for all expressions of $c$ as a sum of primitive tripotents, and is called the rank of the tripotent $c$. 


\subsection{Geometry of the Convex Set $\overline{\mathcal{D}}$}

Recall that the Bergman metric at the origin 0 yields a positive definite Hermitian form $h_{0}$ on $\mathbb{V}$, for which we also use, for convenience, the notation $\langle.,$.$\rangle . The$ associated Hilbert norm is denoted by $\|$.$\| , not to be confused with the spectral$ norm $|$.$| . It is invariant under the action of K$. Two orthogonal tripotents are orthogonal for this inner product, and two primitive idempotents have the same Hilbert norm (here it is necessary to assume that $\mathbb{V}$ is simple).

Let $c$ be a non zero tripotent. As $|c|=1, c$ is in the topological boundary of $\mathcal{D}$. The following proposition gives a rather precise description of the boundary of $\mathcal{D}$ near $c$.

Proposition 29. Let c be a non-zero tripotent in $\mathbb{V}$. Let

$$
\mathbb{H}_{c}=\{x \in \mathbb{V},\langle x, c\rangle=\langle c, c\rangle\}, \quad H_{c}=\{x \in \mathbb{V}, \operatorname{Re}\langle x, c\rangle=\langle c, c\rangle\} .
$$

Then,

$$
\begin{gathered}
\text { for all } x \in \mathcal{D}, \quad|\langle x, c\rangle|<\langle c, c\rangle . \\
\overline{\mathcal{D}} \cap H_{c}=\overline{\mathcal{D}} \cap \mathbb{H}_{c}=c+\left(\overline{\mathcal{D}} \cap \mathbb{V}_{0}(c)\right) .
\end{gathered}
$$

If $c$ is a maximal tripotent, then

$$
\bar{D} \cap H_{c}=\bar{D} \cap \mathbb{H}_{c}=\{c\} .
$$

The convex set $\overline{\mathcal{D}}$ can be further studied by looking at its faces.

Definition 30. Let $\mathcal{C}$ be a closed convex set in a real vector space $E$. A closed convex set $F$ is said to be a face of $\mathcal{C}$ if

$$
c, d \in \mathcal{C}, \quad 0<t<1, t c+(1-t) d \in F \Longrightarrow c, d \in F .
$$

For example, a singleton $\{x\}$, where $x$ is in $\mathcal{C}$, is a face if and only if $x$ is an extremal point of $\mathcal{C}$. A face is said to be proper if it is neither equal to $\mathcal{C}$ nor to $\emptyset$. A proper face is contained in the topological boundary of $\mathcal{C}$.

The intersection of any family of faces is a face. Given a subset $A$ in $\mathcal{C}$, the face generated by $A$ is the smallest face containing $A$, namely the instersection of all faces containing $A$.

Proposition 31. Let $F$ be a proper face of $\overline{\mathcal{D}}$.

i) The real affine span $\langle F\rangle$ of a proper face is automatically a complex affine subspace of $\mathbb{V}$. 
ii) There exists a unique non zero tripotent $c$, such that

$$
F=F(c):=c+\left(\overline{\mathcal{D}} \cap \mathbb{V}_{0}(c)\right)=\langle F\rangle \cap \overline{\mathcal{D}} .
$$

For $c$ a non zero tripotent, the space $\mathbb{V}_{0}(c)$ is a PHJTS (see Proposition 20), and $\mathcal{D} \cap \mathbb{V}_{0}(c)$ is its unit ball for the spectral norm. Hence the interior of the face $F(c)$ relative to $\langle F\rangle$, which is equal to $c+\left(\mathcal{D} \cap \mathbb{V}_{0}(c)\right)$, has a structure of bounded symmetric domain on its own. Its rank, which is also the rank of the PHJTS $\mathbb{V}_{0}(c)$ is called the rank of the face $F(c)$.

Recall that $G$ is the neutral component of the group of holomorphic diffeomorphisms of $\mathcal{D}$. Let $g$ be in $G$. Then the action of $g$ on $\mathcal{D}$ extends to some neighbourhood of $\overline{\mathcal{D}}$ (depending on $g$ ). Hence the action of the group $G$ extends to $\overline{\mathcal{D}}$.

Proposition 32. The group $G$ acts on the set of faces of $\overline{\mathcal{D}}$, preserving the rank of a face. The group $G$ acts transitively on the set of faces of a given rank.

\subsection{The Shilov Boundary of $\mathcal{D}$}

Denote by $S$ be the Shilov boundary of $\mathcal{D}$.

Theorem 33. Let $x$ be in $\mathbb{V}$. Then the following assertions are equivalent:

i) $x$ belongs to $S$

ii) $x$ is an extremal point of the convex set $\overline{\mathcal{D}}$

iii) $x$ is maximal tripotent of the PHJTS $\mathbb{V}$

iv) $\|x\|=\sup \{\|z\| ; z \in \overline{\mathcal{D}}\}$.

Sketch of the Proof of i) $\Longleftrightarrow$ iii). If $z$ is in $\mathcal{D}$, then by Theorem 27 , there exists a Peirce frame $\left(c_{1}, c_{2}, \ldots, c_{r}\right)$ and $0 \leq \lambda_{1} \leq \cdots \leq \lambda_{r}<1$ such that $z=$ $\sum_{j=1}^{r} \lambda_{j} c_{j}$. Let $\mathbb{W}$ be the complex vector space generated by the $c_{j}, 1 \leq j \leq r$, and let $\mathcal{P}$ be the polydisc in $\mathbb{W}$ defined by

$$
\mathcal{P}:=\left\{w=\sum_{j=1}^{r} w_{j} c_{j} ; w_{j} \in \mathbb{C}, \quad\left|w_{j}\right|<1, \quad 1 \leq j \leq r\right\}=\mathbb{W} \cap \mathcal{D} .
$$

The Shilov boundary of the polydisk $\mathcal{P}$ (as a bounded domain in $\mathbb{W}$ ) is the torus

$$
T=\left\{\sigma=\sum_{j=1}^{r} \sigma_{j} c_{j} ;\left|\sigma_{j}\right|=1,1 \leq j \leq r\right\}
$$


(cf Example 1 in Section 4.1). Observe that any element of $T$ is a maximal tripotent of $\mathbb{V}$. Now, if $f$ is a holomorphic function in a neighbourhood $\mathcal{U}$ of $\bar{D}$, then apply the maximum principle to the restriction of $f$ to $\mathbb{W} \cap \mathcal{U}$ (which is a neigbourhood of $\overline{\mathcal{P}}$ ), to get

$$
|f(z)| \leq \sup \{|f(\sigma)|, \quad \sigma \in \mathbb{T}\} \leq \sup \{|f(\sigma)|, \quad \sigma \in \Sigma\}
$$

where $\Sigma$ is the set of all maximal tripotents of $\mathbb{V}$. This shows that the maximum principle holds for $\Sigma$, and hence $S \subset \Sigma$. Conversely, let $c$ be a maximal tripotent. Consider the holomorphic function $f_{c}$ defined by $f_{c}(z)=(2-\langle z, c\rangle)^{-1}$. The function $\zeta \mapsto(2-\zeta)^{-1}$ is holomorphic in a neigbourhood of the closed unit disc $D$ in $\mathbb{C}$ and its modulus has a strict maximum in $\bar{D}$ at $\zeta=1$. Hence the modulus of $f_{c}$ has a strict maximum on $\overline{\mathcal{D}}$ at $z=c$ (use (28)). Hence $c$ must be in the Shilov boundary of $\mathcal{D}$, showing that $\Sigma \subset S$.

As seen earlier, the action of $G$ extends to $\overline{\mathcal{D}}$, and in particular $G$ acts on $S$. The action of $G$ is transitive on $S$, and even the action of $K$ is transitive, a consequence of Proposition 28.

\subsection{The Arithmetic Distance and the $G$-orbits in $S \times S$}

Let $x, y$ be two points in $S$. Then consider the face $\mathcal{F}(x, y)$ generated by $\{x, y\}$. The rank of $\mathcal{F}(x, y)$ is called the arithmetic distance and denoted by $\delta(x, y)$. By Proposition 32, the arithmetic distance is preserved by the action of $G$.

For any $k, 0 \leq k \leq r$, let

$$
\mathcal{O}_{k}:=\{(x, y) \in S \times S ; \delta(x, y)=k\} .
$$

Theorem 34. For any $k, 0 \leq k \leq r, \mathcal{O}_{k}$ is an orbit under $G$. Any orbit of $G$ in $S \times S$ equals $\mathcal{O}_{k}$ for some $k$. Moreover,

$$
\Delta_{S \times S}=\mathcal{O}_{0}=\overline{\mathcal{O}_{0}} \subset \overline{\mathcal{O}_{1}} \subset \cdots \subset \overline{\mathcal{O}_{r}}=\overline{\mathcal{D}} .
$$

The orbit $\mathcal{O}_{r}$ is an open dense subset of $S \times S$. There are useful characterizations of pairs in $\mathcal{O}_{r}$.

Proposition 35. Let $x, y \in S$. The following propositions are equivalent

i) $(x, y)$ belongs to $\mathcal{O}_{r}$.

ii) There is a geodesic ligne $\gamma(t), t \in \mathbb{R}$ in $\mathcal{D}$ such that

$$
\lim _{t \rightarrow-\infty} \gamma(t)=x, \quad \lim _{t \rightarrow+\infty} \gamma(t)=y .
$$


iii) The Bergman kernel $k(z, w)$ defined on $\mathcal{D} \times \mathcal{D}$ can be extended by continuity to $(x, y)$.

A pair $(x, y)$ in $S \times S$ is said to be tranverse (and we then write $x \top y$ ) if any one of these equivalent properties is satisfied.

\subsection{Example (Continued from Sections 2.5 and 3.4)}

We continue notation introduced earlier in the study of $M_{p, q}\left(\right.$ or $\left.\mathcal{D}_{p, q}\right)$.

Assume $p \geq q$. Notice that this is not really a restriction, as by duality, $\mathcal{D}_{p, q} \simeq$ $\mathcal{D}_{q, p}$. But the description of the Shilov boundary is easier in this case. The rank of $M_{p, q}$ is then equal to $q$. Let $s$ be an integer, such that $1 \leq s \leq q$. By an appropriate choice of basis of $\mathbb{W}_{0}$ and $\mathbb{W}_{0}{ }^{\perp}$, a tripotent of rank $s$ can be written as

$$
C_{s}=\left(\begin{array}{lll}
1 & & \\
& \ddots & \\
& & 1 \\
& 0 &
\end{array}\right)
$$

with $s$ times 1 on the main diagonal. The corresponding face is:

$$
\mathcal{F}_{s}=\left\{Z=\left(\begin{array}{ccc}
1 & & \\
& \ddots & \\
& & 1 \\
& \zeta
\end{array}\right)\right\}
$$

where there are $s$ times 1 on the main diagonal and $\zeta$ is an arbitrary element in $\operatorname{Mat}((p-s) \times(q-s), \mathbb{C})$ with $\|\zeta\| \leq 1$.

From the determination of tripotents in Section 3.4, we know that a maximal tripotent $Z$ is obtained for $s=q$, which forces $\mathbb{F}=\mathbb{W}_{0}$. Hence $Z$ is an isometric embedding from $\mathbb{W}_{0}$ into $\mathbb{W}_{0}{ }^{\perp}$. This is equivalent to saying that $Z^{*} Z=I_{q}$. Hence, the Shilov boundary $S$ of the domain $\mathcal{D}_{p, q}$ is given by

$$
S=S_{p, q}=\left\{Z \in \operatorname{Mat}(p \times q, \mathbb{C}) ; Z^{*} Z=I_{q}\right\} .
$$

The space $S_{p, q}$ is called the Stiefel manifold (it can also be considered as the space of all $q$-frames in $\mathbb{C}^{p}$ ). If $p=q, S_{p, p}$ can be identified with the unitary group $\mathrm{U}(p, \mathbb{C})$.

Let $Z$ be in $S$, i.e., $Z$ is an isometric embedding. Then its graph $\mathbb{W}_{Z}$ is a totally isotropic susbspace (i.e., $h_{\mathbb{W}_{Z}}=0$ ) of $\mathbb{E}$. Since $\mathbb{W}_{Z}$ has dimension $q$, it is a 
maximally totally isotropic subspace of $\mathbb{E}$. Conversely, any maximally totally isotropic subspace of $\mathbb{E}$ is of dimension $q$ and can be realized has $\mathbb{W}_{Z}$ for an appropriate $Z$ in $S$. So in the original realization of $M_{p, q}$, the Shilov boundary is realized as the set of all maximally totally isotropic subspaces of $\mathbb{E}$.

Proposition 36. The arithmetic distance on $S$ is given by

$$
\delta\left(Z, Z^{\prime}\right)=\operatorname{rank}\left(Z-Z^{\prime}\right)
$$

Proof: First consider the realization of $S$ as the space of maximally totally isotropic subspaces of $\mathbb{E}$. There is an obvious invariant for the action of $G$ on $S \times S$, namely the dimension of the intersection of the two spaces of the pair. Conversely, let $\mathbb{W}$ and $\mathbb{W}^{\prime}$ be two maximally isotropic subspaces of $\mathbb{E}$, and let $\operatorname{dim}\left(\mathbb{W} \cap \mathbb{W}^{\prime}\right)=s$, with $0 \leq s \leq q$. Then the signature of the restriction of $h$ on $\mathbb{W}+\mathbb{W}^{\prime}$ has to be $(q-s, q-s)$. Hence by Witt's theorem, two such pairs $\left(\mathbb{W}_{1}, \mathbb{W}_{1}^{\prime}\right)$ and $\left(\mathbb{W}_{2}, \mathbb{W}_{2}^{\prime}\right)$ are conjugate under $U(E, h)$ if and only if $\operatorname{dim}\left(\mathbb{W}_{1} \cap \mathbb{W}_{1}^{\prime}\right)=\operatorname{dim}\left(\mathbb{W}_{2} \cap \mathbb{W}_{2}^{\prime}\right)$. This gives a description of the orbits of $G$ in $S \times S$ (cf Theorem 34).

If $\mathbb{W}$ (resp $\mathbb{W}^{\prime}$ ) is realized as the graph of some isometric imbedding $Z$ (respectively $\left.Z^{\prime}\right)$, then $\operatorname{dim}\left(\mathbb{W} \cap \mathbb{W}^{\prime}\right)=\operatorname{dim} \operatorname{ker}\left(Z-Z^{\prime}\right)$. Hence this last quantity is invariant under the action of $G$ on $S \times S$.

Now, consider the following pairs $Z, Z^{\prime}$ of tripotents (one pair in each orbit) namely

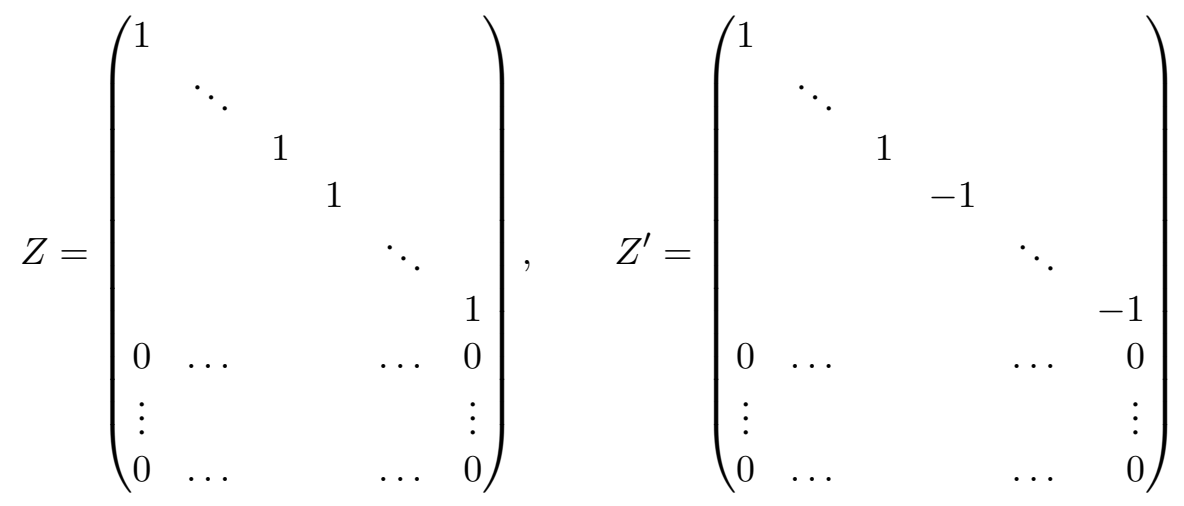

where there are $s$ times 1 (and hence $(q-s)$ times -1 ) on the main diagonal of $Z^{\prime}$. Then, the face generated by $\left\{Z, Z^{\prime}\right\}$ is equal to $\mathcal{F}_{s}$ (see (32)), which is of rank $q-s$. Hence the arithmetic distance $\delta\left(Z, Z^{\prime}\right)$ is equal to $q-s=\operatorname{rank}\left(Z-Z^{\prime}\right)=$ $q-\operatorname{dim} \operatorname{ker}\left(Z-Z^{\prime}\right)$. By the invariance of the latter quantity under the action of $G$, we can conclude that the formula is true for all pairs $\left(Z, Z^{\prime}\right)$. 
Another expression for the arithmetic distance is $\delta\left(Z, Z^{\prime}\right)=\operatorname{rank}\left(I_{q}-Z^{*} Z^{\prime}\right)$. In fact, $Z-Z^{\prime}$ and $I_{q}-Z^{*} Z^{\prime}$ have the same kernel. To see it, first, by left multiplication by $Z^{*}$

$$
\left(Z-Z^{\prime}\right) \xi=0 \Longrightarrow\left(I_{q}-Z^{*} Z^{\prime}\right) \xi=0
$$

and hence, $\operatorname{ker}\left(Z-Z^{\prime}\right) \subset \operatorname{ker}\left(I_{q}-Z^{*} Z^{\prime}\right)$. Conversely,

$$
\left(I_{q}-Z^{*} Z^{\prime}\right) \xi=0 \Rightarrow\left\langle\left(I_{q}-Z^{*} Z^{\prime}\right) \xi, \xi\right\rangle=0 \Rightarrow\left\langle Z^{\prime} \xi, Z \xi\right\rangle=\langle\xi, \xi\rangle .
$$

But $\|Z \xi\|=\left\|Z^{\prime} \xi\right\|=\|\xi\|$ and hence, by Cauchy-Schwartz inequality, $Z^{\prime} \xi=Z \xi$. Hence $\operatorname{ker}\left(Z-Z^{\prime}\right) \supset \operatorname{ker}\left(I_{q}-Z^{*} Z^{\prime}\right)$.

\section{Euclidean Jordan Algebras and Tube-type Domains}

For the theory of Euclidean Jordan algebras, the reader is refered to [13]. A different point of view is presented in [32]. For the rest of this section, we follow mainly [25], Sections 3 and 5.

\subsection{Euclidean Jordan Algebra}

Definition 37. A Euclidean Jordan algebra is a real Euclidean vector space $(W,\langle.,\rangle$. with a bilinear product $(x, y) \mapsto x . y$ and a unit element $e$ such that

i) $x \cdot y=y \cdot x$ (commutativity)

ii) $x^{2} \cdot(x \cdot y)=x \cdot\left(x^{2} \cdot y\right)$ (weak associativity)

iii) $e . x=x . e=x$

iv) $\langle x . y, z\rangle=\langle x, y . z\rangle$ (symmetry property)

for all $x, y, z$ in $W$.

Example. Let $W=\operatorname{Symm}(r, \mathbb{R})$ be the space of $r \times r$ real symmetric matrices and set

$$
x . y=\frac{1}{2}(x y+y x), \quad e=\mathrm{Id}, \quad\langle x, y\rangle=\operatorname{Tr}(x y) .
$$

Then $W$ is a Euclidean Jordan algebra.

Let $W$ be a Jordan Euclidean algebra. For $x$ in $W$, let $P(x)$ be the operator on $W$ defined by

$$
P(x) y=2 x .(x . y)-x^{2} . y .
$$


The map $P$ is called the quadratic representation of the Jordan algebra $W$. For $W=\operatorname{Symm}(r, \mathbb{R}), P(x) y=x y x$.

An element $x$ of $W$ is said to be invertible if $P(x)$ is an invertible operator. Invertible elements form a dense open subset of $W$. If $x$ is an invertible element of $W$, then define its inverse to be $x^{-1}:=P(x)^{-1} x$.

Let $Q:=\left\{x \in W ; y \in W, x=y^{2}\right\}$ be the cone of squares in $W$, and let $\Omega$ be the interior of $Q$. It coincides with the set of squares of invertible elements.

A cone $C$ in a Euclidean vector space $E$ is said to be proper if it does not contain any (affine) line. The dual cone $C^{\sharp}$ is defined by

$$
C^{\sharp}:=\{x \in E ; y \in C, \quad\langle x, y\rangle \geq 0\} .
$$

A cone $C$ is said to be symmetric (or self dual) if its dual $C^{\sharp}$ is equal to its closure $\bar{C}$. The automorphism group of the cone is the subgroup $L=L(C)$ of $\operatorname{GL}(E)$ defined by

$$
L(C):=\{g \in \mathrm{GL}(E) ; g(C)=C\} .
$$

A cone $C$ is said to be homogeneous if its group of automorphisms is transitive on $C$.

Proposition 38. Let $W$ be a Euclidean Jordan algebra. The cone $\Omega$ is convex, proper, symmetric and homogeneous. It is called the symmetric cone of W. Conversely, any convex proper, symmetric and homogeneous cone in a Euclidean space $E$ can be realized as the symmetric cone $\Omega$ for some structure of Euclidean Jordan algebra on $W$.

Example. For $W=\operatorname{Symm}(r, \mathbb{R})$, the symmetric cone $\Omega$ is the cone of positive definite symmetric matrices. The automorphism group of the cone is isomorphic to $\mathrm{GL}(r, \mathbb{R}) / \pm \mathrm{Id}$ acting by $(g, x) \longmapsto g x g^{t}$ for $g$ in $\mathrm{GL}(r, \mathbb{R})$ and $x$ in $\operatorname{Symm}(r, \mathbb{R})$.

Going back to the general case, an element $c$ in a Euclidean algebra $W$ is said to be an idempotent if $c^{2}=c$. Two idempotents $c$ and $d$ are said to be orthogonal if $c . d=0$. Then $c+d$ is an idempotent. A non zero idempotent is said to be primitive if it cannot be written as a sum of two orthogonal nonzero idempotents. Any idempotent can be written as a sum of mutually orthogonal primitive idempotents. A Jordan frame is a set $\left(c_{1}, c_{2}, \ldots, c_{r}\right)$ of mutually orthogonal primitive idempotents such that $e=c_{1}+c_{2}+\cdots+c_{r}$. Two Jordan frames are conjugate under an automorphism of the Jordan algebra $W$. The number of elements in a Jordan frame is called the rank of the Euclidean Jordan algebra $W$. 
Let $x$ be an element in $W$. Then there exists a Jordan frame $\left(c_{1}, c_{2}, \ldots, c_{r}\right)$ and real numbers $\lambda_{1} \leq \lambda_{2} \leq \cdots \leq \lambda_{r}$ such that $x=\sum_{j=1}^{r} \lambda_{j} c_{j}$ (spectral decomposition of $x$ ). The $\lambda_{j}$ 's are unique and called the spectral values of $x$.

There exists a linear form $\operatorname{tr}$ (respectively a homogeneous polynomial det of degree $r$ ) on $V$, called the trace (respectively determinant), such that $\operatorname{tr} x$ (respectively $\operatorname{det} x$ ) is the sum (respectively the product) of its spectral values (counted with multiplicity). An element $x$ is invertible if and only if $\operatorname{det} x \neq 0$.

Example. Let $W=\operatorname{Symm}(r, \mathbb{R})$. Then an idempotent is an orthogonal projector. It is minimal if it is of rank one. A Jordan frame is a complete family of mutually orthogonal projectors of rank one (i.e., associated to an orthonormal basis of $\mathbb{R}^{r}$ ). Spectral values, trace and determinant coincide with the usual notions.

\subsection{Hermitification of a Euclidean Jordan Algebra}

Euclidean Jordan algebras are intimately connected with PHJTS. First, Euclidean Jordan algebras provide examples of PHJTS through the process of Hermitification. Let $W$ be a Euclidean Jordan algebra. Let $\mathbb{W}$ be its complexification. Extend the Jordan product to $\mathbb{W}$ in a $\mathbb{C}$-linear way. On $\mathbb{W}$, define the following triple product

$$
\{x, y, z\}=2(x \cdot(\bar{y} \cdot z)+z \cdot(\bar{y} \cdot x)-\bar{y} \cdot(x \cdot z)) .
$$

Proposition 39. The complex vector space $\mathbb{W}$ with the triple product define by (37) is a PHJTS (called the Hermitification of $W$ ).

An idempotent of $W$ is a tripotent of $\mathbb{W}$, a primitive idempotent of $W$ is a primitive tripotent in $\mathbb{W}$, and a Jordan frame of $W$ is a Peirce frame in $\mathbb{W}$.

Proposition 40. Let $c$ be a maximal tripotent in $\mathbb{W}$. Then there exists a Jordan frame $\left(c_{1}, c_{2}, \ldots, c_{r}\right)$ of $W$ and complex numbers $\left(\lambda_{1}, \lambda_{1}, \ldots, \lambda_{r}\right)$ of modulus 1 such that $c=\sum_{j=1}^{r} \lambda_{j} c_{j}$.

Extend the quadratic representation $P$ from $W$ to $\mathbb{W}$ in a holomorphic way. Similarly, extend the inversion $\left(x \mapsto x^{-1}\right)$ from $W$ to $\mathbb{W}$ in a meromorphic way.

Proposition 41. An element $z$ of $\mathbb{W}$ is a maximal tripotent if and only if $z$ is invertible and satisfies $z^{-1}=\bar{z}$. The Shilov boundary $S$ of the unit ball $\mathbb{D}$ in $\mathbb{W}$ is given by

$$
S=\left\{z \in \mathbb{W} ; z \text { invertible }, z^{-1}=\bar{z}\right\}
$$


In particular, this gives equations for the Shilov boundary $S$ of the unit ball $\mathcal{D}$ in $\mathbb{W}$. The Shilov boundary is a totally real submanifold of $\mathbb{W}$ and

$$
\operatorname{dim}_{\mathbb{R}} S=\operatorname{dim}_{\mathbb{C}} \mathcal{D}
$$

\subsection{Euclidean Jordan Algebra Associated to a Tripotent}

We have the following statement, which (loosely speaking) goes in the opposite direction. Let $\mathbb{V}$ be a PHJTS.

Proposition 42. Let $c$ be a tripotent in $\mathbb{V}$, and let $\mathbb{V}=\mathbb{V}_{2}(c) \oplus \mathbb{V}_{1}(c) \oplus \mathbb{V}_{0}(c)$ be the Peirce decomposition of $\mathbb{V}$ w.r.t. c. Then:

i) the map $z \longmapsto z^{*}:=\frac{1}{2}\{c, z, c\}$ is a conjugate linear involution of $\mathbb{V}_{2}(c)$.

ii) its set of fixed points $W(c):=\left\{z \in \mathbb{V}_{2}(c) ; z^{*}=z\right\}$ is a Euclidean Jordan algebra for the following data:

$$
x . y=\frac{1}{2}\{x, c, y\}, \quad \text { unit } c, \quad\langle x, y\rangle=\operatorname{Re} \tau(x, y) \text {. }
$$

This result (applied for a maximal tripotent) helps to describe the domain $\mathcal{D}$ and its Shilov boundary $S$ near a point $c$ in $S$.

Proposition 43. Let $c$ be a maximal tripotent in $\mathbb{V}$. Let $\mathbb{V}=\mathbb{V}_{2}(c) \oplus \mathbb{V}_{1}(c)$ be the corresponding Peirce decomposition, and let $W(c)$ be the corresponding real form of $\mathbb{V}_{2}(c)$, with its structure of Euclidean Jordan algebra. Let $\Omega(c)$ be the symmetric cone of $W(c)$. Then:

i) the (affine) tangent space $T_{c} S$ of $S$ at $c$ is equal to

$$
T_{c} S=c+\mathrm{i} W(c) \oplus \mathbb{V}_{1}(c)
$$

ii) the following inclusion holds:

$$
\mathcal{D} \subset(c-\Omega(c)+\mathrm{i} W(c)) \oplus V_{1}(c)
$$

See [7] for a proof. 


\subsection{Euclidean Jordan Algebras vs PHJTS}

Let $W$ be a Euclidean Jordan algebra, with unit element $e$, and let $\mathbb{W}$ be its Hermitification. Then $L(e, e)=2 \mathrm{Id}$ and $Q(e)$ is the conjugation of $\mathbb{W}$ with respect to $W$. Hence $e$ is a (maximal) tripotent, the Peirce decomposition with respect to $e$ is trivial (i.e., $\mathbb{W}_{2}(e)=\mathbb{W}$ ), the fixed points set of $Q(e)$ is $W$ and the structure of Euclidean Jordan algebra on $W$ is the initial one.

These properties essentially characterize those PHJTS which can be obtained from a Euclidean Jordan algebra by Hermitification.

Proposition 44. Let $\mathbb{V}$ be a PHJTS. Let $\mathcal{D}$ be its unit ball for the spectral norm of $\mathbb{V}$, and let $S$ be its Shilov boundary. Then the following equivalent properties are equivalent:

i) $\mathbb{V}$ is the Hermitification of some Euclidean Jordan algebra.

ii) If $c$ is a maximal tripotent of $\mathbb{V}$, then the corresponding Peirce decomposition is trivial, i.e., $\mathbb{V}=\mathbb{V}_{2}(c)$.

iii) $S$ is a totally real submanifold of $\mathbb{V}$

iv) $\operatorname{dim}_{\mathbb{R}} S=\operatorname{dim}_{\mathbb{C}} \mathbb{V}$.

Example. The PHJTS $\mathbb{V}=\operatorname{Mat}(p \times q, \mathbb{C})$ is the Hermitification of a Euclidean Jordan algebra if and only if $p=q$. If $p=q$, then $\mathbb{V}$ is the Hermitification of the Euclidean Jordan algebra $\operatorname{Herm}(p, \mathbb{C})$, where the Jordan product is given by $x . y=\frac{1}{2}(x y+y x)$.

\subsection{The Cayley Transform}

Main reference for this section is [25], Section 10. For a presentation of the Cayley transform from the point of view of semisimple Lie groups, see the original paper [34] or [30], Chapter 3.

Let us first give a complement to Proposition 42. We keep the notation from previous sections.

Let $c$ be a tripotent in $\mathbb{V}$. For $a$ be in $\mathbb{V}_{2}(c)$, let $R_{a}$ be the endomorphism of $\mathbb{V}_{1}(c)$ defined by

$$
R_{a}(x)=\{a, c, x\} .
$$

Further, define $\Phi: \mathbb{V}_{1}(c) \times \mathbb{V}_{1}(c) \longrightarrow \mathbb{V}_{2}(c)$ by

$$
\Phi(u, v)=\{u, v, c\} .
$$


Proposition 45. Let $c$ be a tripotent in a PHJT $\mathbb{V}$. Let $R$ and $\Phi$ be defined by (41) and respectively (42). Then:

i) for $a, b$ in $\mathbb{V}_{2}(c)$

$$
\begin{gathered}
\frac{1}{2}\left(R_{a} \circ R_{b}+R_{b} \circ R_{a}\right)=R_{a . b}, \quad R_{e}=\mathrm{Id} \\
R_{a}^{*}=R_{a^{*}}
\end{gathered}
$$

ii) for $a$ is in $\Omega(c)$

$$
R_{a}^{*}=R_{a} \quad \text { and } \quad R_{a} \gg 0 .
$$

iii) $\Phi$ is Hermitian and $\Omega(c)$-positive definite in the sense that, for all $u, v$ in $\mathbb{V}_{1}(c)$

$$
\begin{gathered}
\Phi(u, v)=\Phi(v, u)^{*} \\
\Phi(u, u) \in \overline{\Omega(c)} \text { and } \Phi(u, u)=0 \text { if and only if } u=0 .
\end{gathered}
$$

Recall the notion of Siegel domain of type I and of type II. First, suppose we are given a Euclidean vector space $E$ and a proper open convex cone $\Omega$ in $E$. Let $\mathbb{E}=E+\mathrm{i} E$ be the complexification of $E$. Define $\mathcal{T}=\mathcal{T}(E, \Omega)$ as

$$
\mathcal{T}=\Omega+\mathrm{i} E:=\{z=u+\mathrm{i} v \in \mathbb{E} ; u \in \Omega, v \in E\} .
$$

In other terms, the set $\mathcal{T}$ is the tube over $\Omega$, or the generalized right half-space in $\mathbb{E}$. In our context, it is called the Siegel domain of type I associated to $(E, \Omega)$.

For a Siegel domain of type II, the data are:

i) a Euclidean vector space $E$ with a proper open convex symmetric cone $\Omega$ in $E$

ii) a complex vector space $\mathbb{F}$

iii) a Hermitian and $\Omega$-positive definite map $\Phi: \mathbb{F} \times \mathbb{F} \longrightarrow \mathbb{E}$, where, as usual $\mathbb{E}$ is the complexification of $E$.

Define $\mathcal{S}=\mathcal{S}(E, \Omega, \mathbb{F}, \Phi)$

$$
\mathcal{S}:=\{(u, w) \in \mathbb{E} \times \mathbb{F} ; \operatorname{Re}(u)-\Phi(w, w) \in \Omega\} .
$$

Then $\mathcal{S}$ is called the Siegel domain of type II associated to $(E, \Omega, \mathbb{F}, \Phi)$.

Observe that a Siegel domain of type I is a degenerate case of a Siegel domain of type II (take $\mathbb{F}=\{0\}$ ).

We can now define the Cayley transform. 
Proposition 46. Let $c$ be a maximal tripotent in $\mathbb{V}$. Let $\mathbb{V}=\mathbb{V}_{2}(c) \oplus \mathbb{V}_{1}(c)$ be the Peirce decomposition w.r.t. $c$. For $x$ an arbitrary element of $\mathbb{V}$, let $x=x_{2}+x_{1}$ be the corresponding decomposition of $x$.

i) Let $x$ be in $\mathcal{D}$. Then $c-x_{2}$ is invertible in the complex Jordan algebra $\mathbb{V}_{2}(c)$.

ii) Set, for $x$ in $\mathcal{D}$

$$
\gamma_{c}(x)=\left(e+x_{2}\right)\left(e-x_{2}\right)^{-1}+R_{\left(e-x_{2}\right)^{-1}}\left(x_{1}\right) .
$$

Then $\gamma_{c}$ is a biholomorphic diffeomorphism of $\mathcal{D}$ onto the Siegel domain $\mathcal{S}\left(W(c), \Omega(c), \mathbb{V}_{1}(c), \Phi\right)$.

When the PHJTS $\mathbb{V}$ is the Hermitification of a Euclidean Jordan algebra, then, in our notation $\mathbb{V}_{1}(e)=0$, and the image of $\mathcal{D}$ by the Cayley map is a Siegel domain of type I. The bounded domains corresponding to the PHJTS obtained by Hermitification of a Euclidean Jordan algebra (cf Proposition 44) are called tube type domains.

\section{The Triple Invariant}

The general reference for this section is [11].

Finding invariants is a good tool to study orbits. In this section, we will construct an invariant for the action of $G$ on $S \times S \times S$. We first construct an invariant on $\mathcal{D} \times \mathcal{D} \times \mathcal{D}$, then "pass to the limit" to construct an invariant on $S \times S \times S$. The invariant behaves quite differently wether $\mathcal{D}$ is of tube-type or not.

\subsection{The Symplectic Area of a Geodesic Triangle}

The Kaehler form on $\mathcal{D}$ is the real differential two-form $\omega$ defined on $\mathcal{D}$ by the formula

$$
\omega_{z}(\xi, \eta)=g_{z}\left(\xi, J_{z} \eta\right)
$$

where $\xi$ and $\eta$ are in the tangent space at $z$. The definition as stated is valid on any complex Hermitian manifold. The form $\omega$ is clearly $G$-invariant, and it is a closed form (more generally, this is true for the Kaehler form associated to the Bergman metric of any bounded domain).

Given two points $z, w$ in $\mathcal{D}$, there is a unique geodesic segment starting from $z$ and ending at $w$. This fact is true for any Riemannian symmetric space of the 
noncompact type. Given three points $z_{1}, z_{2}, z_{3}$ in $\mathcal{D}$, one can form the oriented geodesic triangle $T\left(z_{1}, z_{2}, z_{3}\right)$, joining $z_{1}$ to $z_{2}$, then $z_{2}$ to $z_{3}$ and finally from $z_{3}$ to $z_{1}$, each time by using the unique geodesic segment between two summits. Choose a piece of smooth surface $\Sigma$ in $\mathcal{D}$ such that its boundary is the triangle, and orientate $\Sigma$ such that its oriented boundary is $T\left(z_{1}, z_{2}, z_{3}\right)$. Then define the symplectic area of $T\left(z_{1}, z_{2}, z_{3}\right)$ by the formula

$$
A\left(z_{1}, z_{2}, z_{3}\right)=\int_{\Sigma} \omega .
$$

As the form $\omega$ is closed, this integral does not depend on the choice of $\Sigma$ and defines a real valued function on $\mathcal{D} \times \mathcal{D} \times \mathcal{D}$.

It turns out that this function can be explicitely computed. For convenience, we slightly change the normalization of the metric. We will use the metric (proportional to the Bergman metric) which has minimal sectional holomorphic curvature equals to -1 . It amounts to replace, in the defintion of the metric on $\mathcal{D}$ the Bergman kernel $k_{\mathcal{D}}$ by the kernel $k(z, w)=k_{\mathcal{D}}(z, w)^{\frac{2}{p}}$, where $p$ is some integer related to the roots structure of the symmetric space $\mathcal{D}$ (see [11] for details).

Theorem 47. Let $z_{1}, z_{2}, z_{3}$ be three points in $\mathcal{D}$. Then

$$
A\left(z_{1}, z_{2}, z_{3}\right)=-\left(\arg k\left(z_{1}, z_{2}\right)+\arg k\left(z_{2}, z_{3}\right)+\arg k\left(z_{3}, z_{1}\right)\right) .
$$

Observe that $\mathcal{D}$ is simply connected, and for any $z, w$ in $\mathcal{D}, k(z, w) \neq 0$ and $k(z, z)>0$, so that there is a unique continuous determination of the argument of $k(z, w)$ over $\mathcal{D} \times \mathcal{D}$ which takes value 0 on the diagonal.

For (most of) the classical domains, the result is due to Domic and Toledo (see [12]). The computation in the general framework is in [11].

For the unique disc in $\mathbb{C}$, this formula is esssentially equivalent to the classical Gauss formula for the area of a geodesic triangle in the unit disc (with the Poincaré metric) $A=\pi-(\alpha+\beta+\gamma)$, where $\alpha, \beta, \gamma$ are the angles of the triangle.

Proposition 48. The symplectic area satisfies the following properties:

i) $A\left(g\left(z_{1}\right), g\left(z_{2}\right), g\left(z_{3}\right)\right)=A\left(z_{1}, z_{2}, z_{3}\right)$, for all $g$ in $G$.

ii) $A\left(z_{\tau(1)}, z_{\tau(2)}, z_{\tau(3)}\right)=\operatorname{sign}(\tau) A\left(z_{1}, z_{2}, z_{3}\right)$ for $\tau$ any permutation of $\{1,2,3\}$. 
iii) (cocycle property)

$$
A\left(z_{1}, z_{2}, z_{3}\right)=A\left(z_{1}, z_{2}, z_{4}\right)+A\left(z_{2}, z_{3}, z_{4}\right)+A\left(z_{3}, z_{1}, z_{4}\right)
$$

iv) (bounds for the area)

$$
-r \pi<A\left(z_{1}, z_{2}, z_{3}\right)<r \pi
$$

where $z_{1}, z_{2}, z_{3}, z_{4}$ are arbitrary points in $\mathcal{D}$.

Property i) is a consequence of the fact that the defintion of the area uses notions (geodesic triangle, Kaehler form) which are invariant under $G$, ii) reflects the fact that permuting two summits of a triangle changes its orientation, and iii) is a direct consequence of the fact that the Kaehler form is closed (it can also be seen on the formula (46)). The proof of iv) is more subtle and uses the explicit expression given by (46). The bounds are sharp.

\subsection{The Limit Process}

Having contructed this invariant function on $\mathcal{D} \times \mathcal{D} \times \mathcal{D}$, one can "pass to the limit" to construct an invariant on $S \times S \times S$. A triple $\left(\sigma_{1}, \sigma_{2}, \sigma_{3}\right)$ in $S \times S \times S$ is said to be mutually transverse if $\sigma_{i} \top \sigma_{j}$ for $i \neq j$. For mutually transverse triples, the approach to a point of the Shilov boundary is unrestricted.

Theorem 49. Let $\sigma_{1}, \sigma_{2}, \sigma_{3}$ be mutually transverse points in $S$. Then the limit

$$
\iota\left(\sigma_{1}, \sigma_{2}, \sigma_{3}\right)=\frac{1}{\pi} \lim _{z_{j} \rightarrow \sigma_{j}} A\left(z_{1}, z_{2}, z_{3}\right)
$$

exists as $z_{j}$ in $\mathcal{D}$ tends to $\sigma_{j}(j=1,2,3)$.

The proof uses the explicit formula for the symplectic area (46), and the characterization of transverse pairs given in Proposition 35.

For the singular case (at least one pair $\left(\sigma_{i}, \sigma_{j}\right)$ with $i \neq j$ is not transverse), the approach to the Shilov boundary has to be restricted.

Let $c$ be any point in $S$. Then $c$ is a maximal tripotent of $\mathbb{V}$, and the Peirce decomposition of $\mathbb{V}$ with respect to $c$ reads $\mathbb{V}=\mathbb{V}_{2}(c) \oplus \mathbb{V}_{1}(c)$. Let $W(c)$ be the real from of $\mathbb{V}_{2}(c)$ with its structure of Euclidean Jordan algebra and let $\Omega(c)$ be the symmetric cone of $W(c)$ (cf Proposition 42) . Let $\gamma:[0,1] \longrightarrow \mathbb{V}$ be a smooth curve such that $\gamma(0)=c$ and $\gamma(t) \in \mathcal{D}$ for $0<t \leq 1$. By (43), the tangent vector $\dot{\gamma}(0)$ to the curve at $c$ satisfies

$$
\dot{\gamma}(0) \in-\overline{\Omega(c)}+\mathrm{i} W(c) \oplus \mathbb{V}_{1}(c) .
$$


Definition 50. The curve $\gamma$ is said to be $\Omega$-radial at $c$ if

$$
\dot{\gamma}(0) \in-\Omega(c) \oplus \mathbb{V}_{1}(c) .
$$

In other words, there is no restriction on the $\mathbb{V}_{1}$ component of the tangent vector to the curve at $c$ (it allows tangential approach to the Shilov boundary in these directions), but there is a strong condition on its component in $\mathbb{V}_{2}(c)$. For instance, when $\mathbb{D}$ is the unit disc in $\mathbb{C}$ a $\Omega$-radial curve $\gamma$ at the point $c=\gamma(0)$ has to be radial in the usual sense (its derivative at $c$ is perpendicular to the unit circle).

Proposition 51. Let $c$ be in $S$ and let $\gamma$ be a $\Omega$-radial curve at $c$. Let $g$ be in $G$. Then $g \circ \gamma$ is a $\Omega$-radial curve at $g(c)$.

We can now complete Theorem 49 to include non transverse triples.

Theorem 52. Let $\sigma_{1}, \sigma_{2}, \sigma_{3}$ be in $S$. Then the limit

$$
\iota\left(\sigma_{1}, \sigma_{2}, \sigma_{3}\right)=\frac{1}{\pi} \lim _{z_{j} \rightarrow \sigma_{j}} A\left(z_{1}, z_{2}, z_{3}\right)
$$

exists as $z_{j}$ in $\mathcal{D}$ tends to $\sigma_{j}$ along any $\Omega$-radial curve at $\sigma_{j}(j=1,2,3)$. The limit does not depend on the curves used to approach the points $\sigma_{j}$.

For the proof, see [6], [7].

The function $\iota$ will be called the triple invariant on $S$.

Proposition 53. The triple invariant $\iota: S \times S \times S \rightarrow \mathbb{R}$ has the following properties:

i) $\iota\left(g\left(\sigma_{1}\right), g\left(\sigma_{2}\right), g\left(\sigma_{3}\right)\right)=\iota\left(\sigma_{1}, \sigma_{2}, \sigma_{3}\right)$, for all $g$ in $G$

ii) $\iota\left(\sigma_{\tau(1)}, \sigma_{\tau(2)}, \sigma_{\tau(3)}\right)=\operatorname{sign}(\tau) \iota\left(\sigma_{1}, \sigma_{2}, \sigma_{3}\right)$ for any permutation $\tau$ of $\{1,2,3\}$

iii) (cocycle property)

$$
\iota\left(\sigma_{1}, \sigma_{2}, \sigma_{3}\right)=\iota\left(\sigma_{1}, \sigma_{2}, \sigma_{4}\right)+\iota\left(\sigma_{2}, \sigma_{3}, \sigma_{4}\right)+\iota\left(\sigma_{3}, \sigma_{1}, \sigma_{4}\right)
$$

iv) $-r \leq \iota\left(\sigma_{1}, \sigma_{2}, \sigma_{3}\right) \leq r$, for all $\sigma_{1}, \sigma_{2}, \sigma_{3}, \sigma_{4}$ in $S$.

These results are immediate consequences of Proposition 48. The bounds in iv) can be shown to be sharp. There is even a characterization of those triples $\left(\sigma_{1}, \sigma_{2}, \sigma_{3}\right)$ in $S \times S \times S$ for which $\left|\iota\left(\sigma_{1}, \sigma_{2}, \sigma_{3}\right)\right|=r$ (see [11]). 


\subsection{Example: Elie Cartan's Invariant}

Consider the special case of the unit ball in $\mathbb{C}^{2}$ :

$$
\mathcal{D}=\left\{(x, y) \in \mathbb{C}^{2} ; x \bar{x}+y \bar{y}<1\right\} .
$$

On $\mathbb{C}^{3}$, consider the Hermitian form $h$ given by

$$
h\left((z, x, y),\left(z^{\prime}, x^{\prime}, y^{\prime}\right)\right)=z \overline{z^{\prime}}-x \overline{x^{\prime}}-y \overline{y^{\prime}} .
$$

The map $(x, y) \longmapsto \mathbb{C}(1, x, y)$ yields an isomorphism of $\mathcal{D}$ with the open set $\widetilde{\mathcal{D}}$ of the projective space $\mathbb{P}^{2}(\mathbb{C})=\left(\mathbb{C}^{3} \backslash\{0\}\right) / \mathbb{C}^{*}$ defined by

$$
\widetilde{\mathcal{D}}:=\left\{[v] \in \mathbb{P}^{2}(\mathbb{C}) ; h(v, v)>0\right\}
$$

where we have set $[v]=\mathbb{C} v$ for any $v \neq 0$ in $\mathbb{C}^{2}$.

The Shilov boundary of $\mathcal{D}$ (which coincides with its topological boundary in this case) is the unit sphere $S$ in $\mathbb{C}^{2}$. The corresponding boundary of $\widetilde{\mathcal{D}}$ is the $\widetilde{S}$ of isotropic lines (for the form $h$ ) in $\mathbb{C}^{3}$.

The group $\mathrm{PU}(h) \simeq \mathrm{PU}(1,2)$ acts naturally on $\widetilde{\mathcal{D}}$ and on $\widetilde{S}$. These actions can be transferred to $\mathcal{D}$ and $S$ respectively.

In 1932 Elie Cartan (cf. [3]) constructed an invariant for triples of distinct ${ }^{1}$ points in $S$. First observe that if $v$ and $w$ are non proportional isotropic vectors in $\mathbb{C}^{3}$, then $h(v, w) \neq 0$, because otherwise the complex plane generated by $v$ and $w$ would be totally isotropic, which is impossible. Now let $v_{1}, v_{2}, v_{3}$ be three isotropic vectors in $\mathbb{C}^{3} \backslash\{0\}$ mutually non proportional, and consider the complex number

$$
J\left(v_{1}, v_{2}, v_{3}\right)=h\left(v_{1}, v_{2}\right) h\left(v_{2}, v_{3}\right) h\left(v_{3}, v_{1}\right)
$$

which is different from 0 by the previous observation. Now, if we change $v_{j}$ to $\lambda_{j} v_{j}(j=1,2,3)$, then $J$ is multiplied by the factor $\left|\lambda_{1}\right|^{2}\left|\lambda_{2}\right|^{2}\left|\lambda_{3}\right|^{2}$. Hence the $a r$ gument of $J$ depends only on the triple of complex isotropic lines $\left(\left[v_{1}\right],\left[v_{2}\right],\left[v_{3}\right]\right)$. Moreover the principal determination of the argument belongs to $\left[-\frac{\pi}{2},+\frac{\pi}{2}\right]$. In fact, two disctinct points of $S$ are conjugate by an element of $\mathrm{SU}(1,2)$ to (say) the points $(0,-1)$ and $(0,1)$ respectively. If $(x, y)$ is a third point on $S$, then

$$
J((1,-1,0),(1,1,0),(1, x, y))=2\left(|y|^{2}+(x-\bar{x})\right)
$$

proving the claim. Hence arg $J$ gives a well defined invariant on triples of distinct points in $S$, taking values in $\left[-\frac{\pi}{2},+\frac{\pi}{2}\right]$. Up to a factor $\frac{2}{\pi}$, it coincides with the triple invariant $\iota$ we have defined on $S \times S \times S$. For more properties of this invariant, see [15]. For a generalization to the Stiefel manifold, see [4].

\footnotetext{
${ }^{1}$ Observe that two points on $S$ are transverse in the sense of $\mathbf{4 . 5}$ if and only if they are distinct.
} 


\subsection{Example: the Triple Maslov Index}

For a presentation of the classical triple Maslov index, see [23].

Another important example is the celebrated triple Maslov index on the Lagrangian manifold. Let $(E, \omega)$ be a real symplectic vector space of dimension $2 r$. By definition a Lagrangian is a maximal totally isotropic subspace of $E$. The dimension of a Lagrangian is necessarily $r$ and a vector subspace $L$ of dimension $r$ is a Lagrangian if and only if the restriction of $\omega$ to $L \times L$ is identically 0 . The symplectic group $G=\operatorname{Sp}(2 r, \mathbb{R})$ transforms a Lagrangian into another Lagrangian.

The set of all Lagrangians is easily seen to be a closed submanifold of the Grassmannian of $r$-dimensional spaces in $E$, which is called the Lagrangian manifold, denoted by $\Lambda_{r}$. It turns out that it can be realized as the Shilov boundary of a bounded symmetric domain.

Let $W=\operatorname{Symm}(r, \mathbb{R})$ be the Euclidean Jordan algebra of real $r \times r$ symmetric matrices, with Jordan and scalar products

$$
x . y=\frac{1}{2}(x y+y x), \quad\langle x, y\rangle=\operatorname{Tr} x y .
$$

On its Hermitification $\mathbb{W} \simeq \operatorname{Symm}(r, \mathbb{C})$, the spectral norm coincides with the usual operator norm on (symmetric) matrices. Hence the associated bounded symetric domain is the unit ball $\mathcal{D}$ (called the Siegel disc), which can equivalently be defined by

$$
\mathcal{D}:=\left\{z \in \operatorname{Symm}(r, \mathbb{C}) ; 1-z z^{*} \gg 0\right\} .
$$

Let $\mathbb{E}$ be the complexification of $E$, and let $\sigma$ be the conjugation of $\mathbb{E}$ with respect to $E$. Extend $\omega$ as a $\mathbb{C}$-bilinear symplectic form on $\mathbb{E}$. Let $h$ be the Hermitian form on $\mathbb{E} \times \mathbb{E}$ defined by

$$
h\left(\xi, \xi^{\prime}\right)=\frac{\mathrm{i}}{2} \omega\left(\xi, \sigma\left(\xi^{\prime}\right)\right) .
$$

Let $\left(\epsilon_{1}, \ldots, \epsilon_{r}, \phi_{1}, \ldots, \phi_{r}\right)$ be a symplectic basis, i.e., a basis of $E$ such that

$$
\omega\left(\epsilon_{j}, \phi_{j}\right)=-\omega\left(\phi_{j}, \epsilon_{j}\right)=1
$$

for $1 \leq j \leq r$, and 0 for all other pairs of vectors in the basis. Let $e_{j}=\epsilon_{j}+\mathrm{i} \phi_{j}$. Then

$$
h\left(e_{j}, e_{j}\right)=1, \quad h\left(\sigma\left(e_{j}\right), \sigma\left(e_{j}\right)\right)=-1
$$

for $1 \leq j \leq r$ and 0 for all other pairs. Let $\mathbb{V}_{+}$be the complex vector space generated by the $e_{j}, 1 \leq j \leq r$ and let $\mathbb{V}_{-}=\sigma\left(\mathbb{V}_{+}\right)$be the complex vector space 
generated by the $\sigma\left(e_{j}\right), 1 \leq j \leq r$. Then $\mathbb{V}_{+}$and $\mathbb{V}_{-}$are complex Lagrangian subspaces, and

$$
h_{\mid \mathbb{V}_{+} \times \mathbb{V}_{+}} \gg 0, \quad h_{\mid \mathbb{V}_{+} \times \mathbb{V}_{-}}=0, \quad h_{\mid \mathbb{V}_{-} \times \mathbb{V}_{-}} \ll 0 .
$$

Using the basis $\left\{e_{1}, \ldots, e_{r}\right\}$ (respectively $\left\{\sigma\left(e_{1}\right), \ldots, \sigma\left(e_{r}\right)\right\}$ ), identify $\mathbb{V}_{+}$(respectively $\mathbb{V}_{-}$) with $\mathbb{C}^{r}$, and hence $\mathbb{E} \simeq \mathbb{V}_{+} \times \mathbb{V}_{-}$with $\mathbb{C}^{r} \times \mathbb{C}^{r}$. In these setting, $\sigma(\xi, \eta)=(\bar{\eta}, \bar{\xi})$ and the forms $\omega$ and $h$ are given by

$$
h\left((\xi, \eta),\left(\xi^{\prime}, \eta^{\prime}\right)\right)=\xi^{t} \bar{\xi}^{\prime}-\eta^{t} \bar{\eta}^{\prime}, \quad \omega\left((\xi, \eta),\left(\xi^{\prime}, \eta^{\prime}\right)\right)=-2 \mathrm{i}\left(\xi^{t} \eta^{\prime}-\eta^{t} \xi^{\prime}\right)
$$

for $\xi, \xi^{\prime}, \eta, \eta^{\prime} \in \mathbb{C}^{r}$. Let $\widetilde{\mathcal{D}}$ be the set of complex vector subspaces $\mathbb{L}$ of $\mathbb{E}$, of dimension $r$, which satisfy

$$
\omega_{\mid \mathbb{L} \times \mathbb{L}}=0, \quad h_{\mid \mathbb{L} \times \mathbb{L}} \ll 0 .
$$

The set $\widetilde{D}$ is an open set in the complex Lagrangian manifold, which contains $\mathbb{V}$. Let $z$ be in $\operatorname{Mat}(r, \mathbb{C})$. We regard $z$ as an operator from $\mathbb{V}_{-}$into $\mathbb{V}_{+}$and let

$$
\mathbb{L}_{z}=\left\{(z \eta, \eta) ; \eta \in \mathbb{V}_{-}\right\} \subset \mathbb{E}
$$

be its graph. Observe that 0 is mapped to $\mathbb{V}_{-}$.

Proposition 54. The map $z \longmapsto \mathbb{L}_{z}$ is a holomorphic isomorphism of $\mathcal{D}$ onto $\widetilde{\mathcal{D}}$.

The condition $\omega_{\mid \mathbb{L}_{z} \times \mathbb{L}_{z}}=0$ is a consequence of the symmetry of $z$, whereas the condition $h_{\mid \mathbb{L}_{z} \times \mathbb{L}_{z}} \ll 0$ is a consequence of $\|z\|<1$.

Extend in a $\mathbb{C}$-linear way the action of the symplectic group $G$ to $\mathbb{E}$, and observe that $G$ preserves both $\omega$ and $h$. Hence the group $G$ acts on $\widetilde{\mathcal{D}}$, and on $\mathcal{D}$ by transfering the action.

Thanks to Proposition 41, the Shilov boundary $S$ of $\mathcal{D}$ is given by

$$
S=\left\{z \in \operatorname{Symm}(r, \mathbb{C}) ; z z^{*}=1\right\}
$$

If $z$ is in $S$, then its graph $\mathbb{L}_{z}$ satisfies both $\omega_{\mid \mathbb{L} \times \mathbb{L}}=0$ and $h_{\mid \mathbb{L} \times \mathbb{L}}=0$. Such a space $\mathbb{L}$ is stable by $\sigma$, and hence has to be the complexification of some Lagrangian subspace $L$ of $E$. Conversely, the complexification of any Lagrangian $L$ of $E$ can be obtained as the graph of some element in $S$. Hence the Shilov boundary of $\mathcal{D}$ is identified with the Lagrangian manifold $\Lambda_{r}$, in a $G$-equivariant way. 
Let $L_{1}, L_{2}, L_{3}$ be three Lagrangians in $E$. Then, following Kashiwara (see [23]), consider the quadratic form $Q=Q_{L_{1}, L_{2}, L_{3}}$ on $L_{1} \times L_{2} \times L_{3}$ defined by:

$$
Q\left(\xi_{1}, \xi_{2}, \xi_{3}\right)=\omega\left(\xi_{1}, \xi_{2}\right)+\omega\left(\xi_{2}, \xi_{3}\right)+\omega\left(\xi_{3}, \xi_{1}\right)
$$

where $\xi_{1} \in L_{1}, \xi_{2} \in L_{2}, \xi_{3} \in L_{3}$. If $g$ is in $G$, then

$$
Q_{g\left(L_{1}\right), g\left(L_{2}\right), g\left(L_{3}\right)}=Q_{L_{1}, L_{2}, L_{3}} \circ g^{-1}
$$

so that the signature of $Q$ is an invariant under the action of $G$. Define the triple Maslov index of the triple $\left(L_{1}, L_{2}, L_{3}\right)$ by

$$
\iota\left(L_{1}, L_{2}, L_{3}\right)=\operatorname{sign} Q_{L_{1}, L_{2}, L_{3}} .
$$

This defines an invariant (under the action of the symplectic group) for triples of Lagrangians. Through the identification of the Lagrangian manifold with the Shilov boundary of the Siegel disc, the triple Maslov index coincides with the triple invariant on $S \times S \times S$ which we have defined in Theorem 52 .

For all classical domains of tube-type, there is an analog of Kashiwara's formula for the triple invariant (see [5]). The situation is specially interesting for the domain corresponding to the Euclidean Jordan algebras of rank two (type IV).

\section{G-orbits in $S \times S \times S$ (Tube-type Case)}

The reference for this section is [9].

There is a great difference between our two examples. In the case of the unit sphere in $\mathbb{C}^{2}$ (which is the Shilov boundary a non tube-type domain of rank 1), the triple index takes all values in the interval $[-1,1]$. In the case of the Lagrangian manifold $\Lambda_{r}$ (which is the Shilov boundary of a tube-type domain of rank $r$ ), the triple index has values in the set of integers $\{-r,-r+1, \ldots, r-1, r\}$. This is characteristic of the difference between tube-type domains and non-tube type domains. This reflects a qualitative difference in the orbit picture of $G$ in $S \times S \times S$. In the non-tube-type case, there is a continuous family of $G$-orbits, whereas in the tube-type case, there is only a finite number of $G$ - orbits. In the latter case, one can even give a classification of the orbits. Let us present some more details for the tube type case.

So, let $\mathcal{D}$ be a bounded symmetric domain of tube-type, realized as the unit ball in a PHJTS $\mathbb{W}$ which is the Hermitification of some Euclidean Jordan algebra $W$. Let $S$ be its Shilov boundary. 
Proposition 55. The Shilov boundary $S$ has a natural structure of compact Riemannian symmetric space, for which the group $K$ acts by isometries.

Sketch of the proof (see [25]) . Let $c$ be a point in $S$, i.e., $c$ is a maximal tripotent in $\mathbb{W}$. The Peirce decomposition w.r.t. $c$ is just $\mathbb{W}=\mathbb{W}_{2}(c)$, and $Q(c)$ is a (conjugate-linear) involution of $\mathbb{W}$. Its set of fixed points $W(c)$ has a structure of Euclidean Jordan algebra, isomorphic to $W$. The tangent space $T_{c} S$ of $S$ at $c$ can be identified with $\mathrm{i} W(c)$, and one can transport the invariant inner product on the Euclidean Jordan algebra $W(c)$ to define an inner product on $T_{c} S$. As $c$ varies, this defines a Riemannian structure on $S$, which is invariant under $K$. Moreover, $Q(c)$ maps $S$ into itself, yielding an involutive isometry of $S$. But $Q(c)$ acts on $T_{c} S \simeq \mathrm{i} W(c)$ by -1 , and hence coincides with the geodesic symmetry at $c$.

Define a torus to be a maximal flat submanifold in $S$. One way of obtaining a torus is to use a Jordan frame $\left(c_{1}, c_{2}, \ldots, c_{r}\right)$ in $W$. Then

$$
T:=\left\{z=\sum_{j=1}^{r} \mathrm{e}^{\mathrm{i} \theta_{j}} c_{j} ; \theta_{j} \in \mathbb{R} / 2 \pi \mathbb{Z}\right\}
$$

is a torus in $S$.

Let $T$ be a torus in $S$. Then, given any couple $(x, y)$ in $S \times S$, there exists an element $k$ in $K$ such that $k x$ and $k y$ belong to $T$ (an important result in the theory of compact Riemannian symmetric spaces, see [17], Chapter VII).

Theorem 56. Let $T$ be a torus in $S$. Let $x, y, z$ be three points in $S$. There exists an element $g$ of $G$ such that $g(x), g(y), g(z)$ belong to $T$.

In other words, any $G$-orbit in $S \times S \times S$ meets $T \times T \times T$. This result is very helpful towards the classification of $G$-orbits.

To give the classification result, we need one last invariant on $S \times S \times S$. Let $x, y, x$ be three points in $S$. Form the face $\mathcal{F}_{x, y, z}$ generated by the subset $\{x, y, z\}$, and define $\delta(x, y, z)$ to be the rank of $\mathcal{F}_{x, y, z}$. Then clearly, $\delta(x, y, z)$ is invariant under the action of $G$. Notice that this invariant is symmetric with respect to permutations of the three points.

Theorem 57. Let $x, y, z$ (respectively $x^{\prime}, y^{\prime}, z^{\prime}$ ) be in $S \times S \times S$. Then there exists an element $g$ of $G$ such that $x^{\prime}=g(x), y^{\prime}=g(y), z^{\prime}=g(z)$ if and only if

$$
\begin{gathered}
\delta(x, y)=\delta\left(x^{\prime}, y^{\prime}\right), \quad \delta(y, z)=\delta\left(y^{\prime}, z^{\prime}\right), \quad \delta(z, x)=\delta\left(z^{\prime}, x^{\prime}\right) \\
\delta(x, y, z)=\delta\left(x^{\prime}, y^{\prime}, z^{\prime}\right), \quad \iota(x, y, z)=\iota\left(x^{\prime}, y^{\prime}, z^{\prime}\right) .
\end{gathered}
$$


In other words, the five invariants (the three mutual arithmetic distances, the rank $\delta$ of the face generated by the three points and the triple index $\iota$ ) characterize the $G$-orbits. Notice in particular that it implies that there is only a finite number of $G$-orbits in $S \times S \times S$. Fixing a torus $T$ in $S$ (or a Jordan frame in $W$ ), it is possible to give a representative in $T \times T \times T$ of each $G$-orbit in $S \times S \times S$. The five invariants are not quite independant (for instance, an obvious inequality is $\delta(x, y) \leq \delta(x, y, z)$ ), but one can give precisely the conditions on the values of these invariants in order to have a corresponding $G$-orbit (see [9] for details). For the case of the Lagrangian manifold, the classification of the orbits of $\operatorname{Sp}(2 r, \mathbb{R})$ into $\Lambda_{r} \times \Lambda_{r} \times \Lambda_{r}$ is in [19].

\section{The Maslov Index for Paths}

The main reference for this section is [8].

In symplectic geometry, the theory of the triple Maslov index is only one aspect of the theory of the Maslov index. There are other indices, more or less related to the triple Maslov index. Each of them can be generalized in the context of Shilov boundaries of bounded symmetric domains of tube-type. We will concentrate on the generalization of the Maslov index for a path of Lagrangians. From our point of view (which does not follow the historical development of these notions), it arises naturally in relation to the cocycle property of the triple invariant, when addressing the question of the existence of a primitive for this cocycle.

Use notation of Section 7. Let $m$ be a $\mathbb{Z}$-valued function on $S \times S$ which has the following properties:

$$
m(g(x), g(y))=m(x, y), \quad m(y, x)=-m(y, x)
$$

for $x, y$ in $S \times S$ and $g$ in $G$.

Then it is easily verified that the function

$$
\mu(x, y, z)=m(x, y)+m(y, z)+m(z, x)
$$

on $S \times S \times S$ is $\mathbb{Z}$-valued and has the following properties:

i) $\mu(g(x), g(y), g(z))=\mu(x, y, z)$ for $x, y, z$ in $S$ and $g$ in $G$

ii) $\mu$ is skew-symetric with respect to permutations of $\{x, y, z\}$

iii) $\mu(x, y, z, t)=\mu(x, y, t)+\mu(y, z, t)+\mu(z, x, t)$, for all $x, y, z, t$ in $S$. 
The function $m$ is called a primitive of $\mu$. The attentive reader will observe that this has a cohomological flavor, which will not be discussed here (for more information, see [16] and [27]).

There exists no primitive for the triple invariant $\iota$ on $S \times S \times S$, i.e., no function $m$ on $S \times S$ satisfying the asumptions (52), and which would satisfy (53) for $\mu=\iota$. But there is in some sense a substitute, by going to the universal cover of $S$. In fact $S$ is not simply connected.

Proposition 58. Let $\mathcal{D}$ be an irreducible bounded symmetric domain of tube-type, and let $S$ be its Shilov boundary. Then $\pi_{1}(S)$, the first homotopy group of $S$, is isomorphic to $\mathbb{Z}$.

Recall that the Shilov boundary of the unit ball of $\mathbb{W}$ is

$$
S=\left\{z \in \mathbb{W} ; \bar{z}=z^{-1}\right\} .
$$

If $z$ is in $S$, then $(\operatorname{det} z)^{-1}=\operatorname{det}\left(z^{-1}\right)=\operatorname{det} \bar{z}=\overline{\operatorname{det} z}$, so that $|\operatorname{det} z|=1$. Let $S_{1}=\{z \in S$; $\operatorname{det} z=1\}$. Then $S_{1}$ is simply connected and the universal covering of $S$ can be realized as $\widetilde{S}$, where

$$
\widetilde{S}:=\left\{(z, \theta) \in S_{1} \times \mathbb{R} ; \operatorname{det} z=\mathrm{e}^{\mathrm{i} r \theta}\right\} .
$$

Then there exists a $\mathbb{Z}$-valued function $\widetilde{m}$ on $\widetilde{S} \times \widetilde{S}$ which is a primitive of the triple Maslov index $\iota$, in the sense that:

i) $\widetilde{m}$ is invariant by the diagonal action of (some covering of) $G$

ii) $\widetilde{m}$ is invariant by the diagonal action of $\pi_{1}(S)$, i.e., $m(T \widetilde{x}, T \widetilde{y})=m(\widetilde{x}, \widetilde{y})$ for any $T$ in $\pi_{1}(S)$

iii) $\widetilde{m}$ is skew-symmetric, i.e., $\widetilde{m}(\widetilde{x}, \widetilde{y})=-\widetilde{m}(\widetilde{y}, \widetilde{x})$

iv) for any three points $\widetilde{x}_{1}, \widetilde{x}_{2}, \widetilde{x}_{3}$ in $\widetilde{S}$, the sum

$$
\widetilde{m}\left(\widetilde{x}_{1}, \widetilde{x}_{2}\right)+\widetilde{m}\left(\widetilde{x}_{2}, \widetilde{x}_{3}\right)+\widetilde{m}\left(\widetilde{x}_{3}, \widetilde{x}_{1}\right)
$$

depends only on the projections $x_{1}, x_{2}, x_{3}$ of $\widetilde{x}_{1}, \widetilde{x}_{2}, \widetilde{x}_{3}$ on $S$ and is equal to $\iota\left(x_{1}, x_{2}, x_{3}\right)$.

The construction of $\widetilde{m}$ follows the original construction proposed by Souriau for the Lagrangian manifold (see [31] or [16]).

Notice that a function on $\widetilde{S} \times \widetilde{S}$, which is invariant by the diagonal action of $\pi_{1}(S)$ is nothing but a function defined for paths in $S$, which is invariant under 
a homotopy of the path (with fixed extremities). This is the point of view in the original and more geometric approach, due to Maslov, Arnold and Leray for the Lagrangian manifold, leading to the notion of the Maslov index for a path of Lagrangians. We sketch a presentation of the generalization of this approach (see [8] for details).

Fix $x_{0}$ a point in $S$, and define the Maslov cycle based at $x_{0}$ as the set $\Sigma\left(x_{0}\right)$ defined by

$$
\Sigma\left(x_{0}\right)=S \backslash\left\{x \in S ; x \top x_{0}\right\} .
$$

As the point $x_{0}$ is supposed to be fixed, we drop the index $x_{0}$. The set $\Sigma=\Sigma\left(x_{0}\right)$ is a (real) algebraic hypersurface, as

$$
\Sigma=\left\{x \in S ; \operatorname{det}\left(x-x_{0}\right)=0\right\} .
$$

It admits the following stratification:

$$
\Sigma=\bigsqcup_{j=1}^{r} \Sigma^{(j)}, \quad \Sigma^{(j)}=\left\{x \in S ; \delta\left(x, x_{0}\right)=r-j\right\} .
$$

By computing the codimension of each stratum $\Sigma^{(j)}$ in $S$, it can be shown that the singular set of $\Sigma$ (which is equal to $\sqcup_{j=2}^{r} \Sigma^{(j)}$ ) has codimension at least three in $S$, the regular stratum $\Sigma^{(1)}$ being an (open) hypersurface in $S$.

Let $x$ be a point in $\Sigma^{(1)}$. The tangent space $T_{x} S$ of $S$ at $x$ has a natural structure of Euclidean Jordan algebra, and in particular, there is a symmetric cone $\Omega_{x}$ in it. It turns out that $H_{x}$, the tangent hyperplane to $\Sigma^{(1)}$ at $x$ does not meet the cone $\Omega_{x}$. Hence, as the cone $\Omega_{x}$ is convex, it lies entirely inside one open half-space limited by $H_{x}$. A transverse orientation of the Maslov cycle is obtained at each regular point $x$ of $\Sigma$ (i.e., $x \in \Sigma^{(1)}$ ) by declaring positive the half-space of $T_{x} S$ limited by $H_{x}$ that contains the cone $\Omega_{x}$.

These two geometric properties of $\Sigma$ (the singular set of $\Sigma$ is of codimension greater than or equal to $3, \Sigma$ admits a transverse orientation) are the key ingredients in the construction of a $\mathbb{Z}$-valued index for paths, due to Arnold (see [1]).

Definition 59 (admissible path) Let $\gamma(t), 0 \leq t \leq 1$ be a path in $S$, with endpoints $x=\gamma(0)$ and $y=\gamma(1)$ not in $\Sigma$. The path is said to be admissible if the following conditions are satisfied:

i) $\gamma$ is a smooth map

ii) $\gamma(t)$ does not belong to $\Sigma$ except for a finite number of values of $t$, say $t_{1}, t_{2}, \ldots, t_{l}$ in the increasing order 
iii) for each $j, 1 \leq j \leq l, x_{j}=\gamma\left(t_{j}\right)$ belongs to $\Sigma^{(1)}$ and the tangent vector $\dot{\gamma}\left(t_{j}\right)$ of the path at $x_{j}$ is transverse to $\Sigma^{(1)}$.

Let $\gamma$ be an admissible path. For each $j, 1 \leq j \leq l$, let $\epsilon_{j}$ be +1 if the tangent vector $\dot{\gamma}\left(t_{j}\right)$ belongs to the positive half-space limited by $H_{x_{j}}$, and $\epsilon_{j}=-1$ if not. Now define the Maslov index of the path $\gamma$ (relative to $x_{0}$ ) to be

$$
\operatorname{Mas}_{x_{0}}(\gamma)=\sum_{j=1}^{l} \epsilon_{j} .
$$

Theorem 60. Let $x$ and $y$ be two points in $S$, not belonging to the Maslov cycle $\Sigma\left(x_{0}\right)$. Then:

i) any homotopy class of paths with origin $x$ and end $y$ contains an admissible path.

ii) two admissible paths with origin $x$ and end $y$ which are homotopic have the same Maslov index.

The theorem allows to extend the defintion of the Maslov index to arbitrary paths, provided their extremities do not belong to the Maslov cycle based at $x_{0}$.

The Maslov index of a path depends on the point $x_{0}$. It is however possible to construct from it a function on $\widetilde{S} \times \widetilde{S}$, which has a simple relation to the primitive constructed à la Souriau (see again [8] for details).

The Maslov index has many applications in mathematics and in mathematical physics (metaplectic representation, geometrical optics, semiclassical approximation to quantum mechanics). See [2], [16], [22], [23], [26].

\section{Appendix: List of Bounded Symmetric Domains and their Shilov Boundaries}

We give the list of the simple bounded symmetric domains and the list of the Shilov boundaries of the simple tube type domains. Notations for Lie groups and Lie algebras are those of [17]. The classification can be obtained either by first classifying the simple Riemannian symmetric spaces, then looking for those cases where $\mathfrak{k}$ has a non trivial center (this is the approach in [17]) or one can classify the PHJTS (see [25], Section 4). 


\section{List of simple bounded symmetric domains}

\begin{tabular}{|c|c|c|c|c|}
\hline & $\mathbb{V}$ & $\mathfrak{g}$ & tube type & rank \\
\hline I & $\operatorname{Mat}(p \times q, \mathbb{C})$ & $\mathfrak{s u}(p, q)$ & yes if $p=q$ & $\inf (p, q)$ \\
\hline II & $\operatorname{Skew}(n, \mathbb{C})$ & $\mathfrak{s o}^{*}(2 n, \mathbb{C})$ & yes if $n$ even & {$\left[\frac{n}{2}\right]$} \\
\hline III & $\operatorname{Symm}(n, \mathbb{C})$ & $\mathfrak{s p}(2 n, \mathbb{R}$ & yes & $n$ \\
\hline IV & $\mathbb{C} \times \mathbb{C}^{n-1}$ & $\mathfrak{s o}(2, n)$ & yes & 2 \\
\hline V & $\operatorname{Mat}(1 \times 2, \mathbb{O})$ & $\mathfrak{e}_{6,(-14)}$ & no & 2 \\
\hline VI & $\operatorname{Herm}(3, \mathbb{O})$ & $\mathfrak{e}_{7,(-25)}$ & yes & 3 \\
\hline
\end{tabular}

N.B. $\mathbb{V}$ stands for the corresponding PHJTS, and $\mathfrak{g}$ is the Lie algebra of the group of holomorphic diffeomorphisms of the domain.

\section{List of Shilov boundaries of bounded symmetric domains of tube type}

\begin{tabular}{|c|c|c|}
\hline$W$ & $\mathrm{G}$ & $\mathrm{S}$ \\
\hline $\operatorname{Symm}(n, \mathbb{R})$ & $\mathrm{Sp}(2 n, \mathbb{R})$ & $\Lambda_{n} \simeq \mathrm{U}(n, \mathbb{C}) / \mathrm{O}(n, \mathbb{R})$ \\
\hline $\operatorname{Herm}(n, \mathbb{C})$ & $\mathrm{SU}(n, n)$ & $\mathrm{U}(n, \mathbb{C})$ \\
\hline $\operatorname{Herm}(n, \mathbb{H})$ & $\mathrm{SO}^{*}(4 n)$ & $\mathrm{U}(2 n, \mathbb{C}) / \mathrm{SU}(n, \mathbb{H})$ \\
\hline $\mathbb{R}^{1, n-1}$ & $\mathrm{SO}_{0}(2, n)$ & $\mathrm{S}^{1} \times \mathrm{S}^{n-1} / \mathbb{Z}_{2}$ \\
\hline $\operatorname{Herm}(3, \mathbb{O})$ & $E_{7,(-25)}$ & $\mathrm{U}(1) E_{6} / F_{4}$ \\
\hline
\end{tabular}

NB. $W$ stands for the Euclidean Jordan algebra in the complexification of which the tube type domain is realized, $G$ is (up to a finite covering) the neutral component of the group of holomorphic diffeomorphisms of the domain, and $S$ is its Shilov boundary.

\section{References}

[1] Arnol'd V., On a Characteristic Class Entering into Conditions of Quantization, Funk. Anal. i Prilozen 1 (1967) 1-14.

[2] Cappell S., Lee R. and Miller E., On the Maslov Index, Comm. Pure and Appl. Math. XVII (1994) 121-183.

[3] Cartan E., Sur le Groupe de la Géométrie Hypersphérique, Comm. Math. Helv. 4 (1932) 158-171. 
[4] Clerc J-L., A Triple Ratio on the Unitary Stiefel Manifold, L'Enseignement Mathématique 48 (2002) 51-71.

[5] Clerc J-L., The Maslov Triple Index on the Shilov Boundary of a Classical Domain, J. Geom. Physics 49 (2004) 21-51.

[6] Clerc J-L., L’indice de Maslov Généralisé, J. Math. Pures Appl. 83 (2004) 99-114.

[7] Clerc J-L., An Invariant for Triples in the Shilov Boundary of a Bounded Symmetric Domain, Comm. Anal. Geom. 15 (2007) 147-173.

[8] Clerc J-L. and Koufany K., Primitive du Cocycle de Maslov Généralisé, Math. Ann. 337 (2007) 91-138.

[9] Clerc J-L. and Neeb K-H., Orbits of Triples in the Shilov Boundary of a Bounded Symmetric Domain, Transformation Groups 11 (2006) 387-426.

[10] Clerc J-L. and Ørsted B., The Maslov Index Revisited, Transformation Groups 6 (2001) 303-320.

[11] Clerc J-L. and Ørsted B., The Gromov Norm of the Kaehler Class and the Maslov Index, Asian J. Math. 7 (2003) 269-296.

[12] Domic A. and Toledo D., The Gromov Norm of the Kaehler Class of Symmetric Domains, Math. Ann. 276 (1987) 425-432.

[13] Faraut J. and Korányi A., Analysis on Symmetric Cones, Oxford Mathematical Monographs, Clarendon Press, Oxford, 1994.

[14] Faraut J. et al. Analysis and Geometry on Complex Homogeneous Domains, Part III Fonctions Spaces on Bounded Symmetric Domains by A. Korányi, Progress in Mathematics 185, Birkäuser, Boston, 2000.

[15] Goldman W., Complex Hyperbolic Geometry, Oxford Math. Monographs, Clarendon Press, Oxford, 1999.

[16] Guillemin V. and Sternberg S., Geometric Asymptotics, Math. Surveys 14, Am. Math. Soc., Providence, 1977.

[17] Helgason S., Differential Geometry, Lie Groups and Symmetric Spaces, Academic Press, Toronto, 1978.

[18] Hua L., Harmonic Analysis of Functions of Several Complex Variables in the Classical Domains, Peking, 1958.

[19] Kashiwara M. and Schapira P., Sheaves on Manifolds, Grundlehren des Math. Wissen. 292, Springer, New York, 1990.

[20] Knapp A., Lie Groups Beyond an Introduction, Birkhäuser, Boston, 1996.

[21] Koecher M., An Elementary Approach to Bounded Symmetric Domains, Rice University, Houston, 1969. 
[22] Leray J., Analyse Lagrangienne et Mécanique Quantique, Séminaire sur les Equations aux Dérivées partielles (1976-1977), Exp. 1, Collège de France, Paris, 1977.

[23] Lion G. and Vergne M., The Weil Representation, Maslov Index and Theta Series, Progress in Mathematics, Birkhäuser, 1980.

[24] Loos O., Symmetric Spaces, I\&II, Benjamin, New York,1969.

[25] Loos O., Bounded Symmetric Domains and Jordan Pairs, Mathematical Lectures, University of California at Irvine, 1977.

[26] Maslov V., Théorie des Perturbations et Méthodes Asymptotiques, Dunod, Paris, 1972.

[27] Monod N., Continuous Bounded Cohomology of Locally Compact Groups, LNM vol. 1758, Springer, Berlin, 2001

[28] Myers S. and Steenrod N., The Group of Isometries of a Riemannian Manifold, Ann. of Math. 40 (1939) 400-416.

[29] Piatetsky-Shapiro I., Géométrie des Domaines Classiques et Théorie des Fonctions Automorphes, Dunod, Paris, 1966.

[30] Satake I., Algebraic Structures of Symmetric Domains, Kan Memorial Lectures 4, Iwanami Shoten and Princeton University Press, 1980.

[31] Souriau J-M., Construction Explicite de l'Indice de Maslov. Applications, Lectures Notes in Physics 50, Springer, Berlin, 1976, pp 117-148.

[32] Springer A., Jordan Algebras and Algebraic Groups, Erg. Math. 75, Springer, New York, 1973.

[33] Vigué J-P., Le Groupe des Automorphismes Analytiques d'un Domaine Borné Symétrique d'un Espace de Banach Complexe. Applications aux Domaines Bornés Symétriques, Ann. Sci. Ec. Norm. Sup. 9 (1976) 202-282.

[34] Wolf J. and Korányi A., Realization of Hermitean Symmetric Spaces as Generalized Half-planes, Ann. Math. 81 (1965) 265-288.

Jean-Louis Clerc

Institut Elie Cartan

Nancy-Université, CNRS, INRIA

Campus des Aiguillettes, BP 239

54506 Vandœuvre-lès-Nancy

FRANCE

E-mail address: jlclerc@iecn.u-nancy.fr 